Mahmoudi, Y., Karimi, N., Deiterding, R., and Emami, S. (2014) Hydrodynamic instabilities in gaseous detonations: comparison of Euler, Navier-Stokes, and large-eddy simulation. Journal of Propulsion and Power, 30 (2). pp. 384-396. ISSN 0748-4658

Copyright (C) 2013 by the American Institute of Aeronautics and Astronautics, Inc.

A copy can be downloaded for personal non-commercial research or study, without prior permission or charge

Content must not be changed in any way or reproduced in any format or medium without the formal permission of the copyright holder(s)

When referring to this work, full bibliographic details must be given

$\underline{\text { http://eprints.gla.ac.uk/92658 }}$

Deposited on: 20 March 2014

Enlighten - Research publications by members of the University of Glasgow http://eprints.gla.ac.uk 


\title{
Hydrodynamic Instabilities in Gaseous Detonations: Comparison of Euler, Navier-Stokes, and Large-Eddy Simulation
}

\author{
Y. Mahmoudi* \\ Delft University of Technology, 2628 CD Delft, The Netherlands \\ N. Karimi \pm \\ University of Cambridge, Cambridge, England CB2 1TN, United Kingdom \\ R. Deiterding \\ Oak Ridge National Laboratory, Oak Ridge, Tennessee 37831 \\ and \\ S. Emami $\stackrel{\S}{-}$ \\ Tarbiat Modares University, XXXX Tehran, Iran \\ DOI: $\underline{10.2514 / 1 . B 34986}$
}

\begin{abstract}
A large-eddy simulation is conducted to investigate the transient structure of an unstable detonation wave in two dimensions and the evolution of intrinsic hydrodynamic instabilities. The dependency of the detonation structure on the grid resolution is investigated, and the structures obtained by large-eddy simulation are compared with the predictions from solving the Euler and Navier-Stokes equations directly. The results indicate that to predict irregular detonation structures in agreement with experimental observations the vorticity generation and dissipation in small scale structures should be taken into account. Thus, large-eddy simulation with high grid resolution is required. In a low grid resolution scenario, in which numerical diffusion dominates, the structures obtained by solving the Euler or Navier-Stokes equations and large-eddy simulation are qualitatively similar. When high grid resolution is employed, the detonation structures obtained by solving the Euler or Navier-Stokes equations directly are roughly similar yet equally in disagreement with the experimental results. For high grid resolution, only the large-eddy simulation predicts detonation substructures correctly, a fact that is attributed to the increased dissipation provided by the subgrid scale model. Specific to the investigated configuration, major differences are observed in the occurrence of unreacted gas pockets in the high-resolution Euler and Navier-Stokes computations, which appear to be fully combusted when large-eddy simulation is employed.
\end{abstract}

\section{Introduction}

A DETONATION consists of a shock wave coupled with a reaction zone propagating at supersonic speed. The leading shock propagates either as a Mach stem or an incident wave. Transverse shocks move laterally behind the front and form triple points at the junction of the incident shock, Mach shock, and transverse wave [1]. Shear layers originate from the triple points. These shear layers separate gas streams that have passed through portions of the leading shock with different strengths, often separating reacted and partly reacted gases $[1,2]$. Despite the complex nature of detonation propagation, it is well known that the shock compression dominates the ignition mechanism in low activation energy mixtures, which are characterized by their laminar reaction zones [3,4]. Nevertheless, experiments have exhibited the occurrence of two types of detonation structure corresponding to two different types of flow behind the main shock [4]. Schlieren visualizations and numerical simulations of the reaction zone structures in irregular structure detonations indicate that the shock front cannot compress all of the inflow [5-9]. Hence, pockets of the unreacted gas exist behind the front [5-9]. In regular structure detonations, intense chemical activity is observed behind the leading shocks, where very few signs of unreacted pockets are found [9-11]. Conversely, it has

Received 10 March 2013; revision received 24 June 2013; accepted for publication 3 July 2013; published online XX epubMonth XXXX. Copyright (C) 2013 by the American Institute of Aeronautics and Astronautics, Inc. All rights reserved. Copies of this paper may be made for personal or internal use, on condition that the copier pay the $\$ 10.00$ per-copy fee to the Copyright Clearance Center, Inc., 222 Rosewood Drive, Danvers, MA 01923; include the code 1533-3876/YY and $\$ 10.00$ in correspondence with the CCC.

*Department of Process and Energy, Mekelweg 2; s.y.mahmoudilarimi@ tudelft.nl (Corresponding Author).

Department of Engineering.

P.O. Box 2008, MS 6367.

${ }^{\S}$ Department of Mechanical Engineering. been shown numerically [1ㅡ] and experimentally $[\underline{4}, \underline{13}-\underline{-15}]$ that in irregular structure detonations hydrodynamic instabilities generate a turbulent mixing zone of hot reacted gases and cold unreacted materials. These instabilities are of Richtmyer-Meshkov (RM) and Kelvin-Helmholtz (KH) types [15-17]. Compressible turbulence in detonation may be generated by various interactions such as shockshock, shock-shear-layer, shock-vortex, and shear-layer-shearlayer interactions. Lee [18] argued that this enhances turbulent mixing in unstable detonations and supplements the shock ignition mechanism.

Despite decades of research on the detonation phenomenon, our general understanding of unstable detonation propagation is still highly incomplete [19]. For instance, the state of a turbulent reaction zone and the consumption mechanism of unreacted gas pockets have remained mostly unexplored $[1,19,20]$. It has been shown $[4,12,15,16,18,21]$ that in strongly unstable detonations diffusive turbulent mixing plays a key role in propagation. However, in weakly unstable detonations the main shock front is responsible for the initiation of the reaction and turbulent phenomena are of minor importance. In particular, it was found that in strongly unstable detonations some portions of the gases typically pass through the main shock without reacting $[12,19]$. Further, for a detonation to propagate in a self-sustained manner and not to fail outright, turbulent mixing and diffusive reaction must occur behind the main front $[15,16,22]$.

Unlike classical turbulence, detonations may display a double energy cascade, a first one from small scales upward and a second from large scales downward [7]. Characterization of such nonequilibrium turbulence and establishment of its relevance to classical turbulence are substantial tasks in detonation research [7]. Importantly, the flow is constantly affected by shock-shear-layer interactions. It therefore appears that the time scales in a detonation are usually not sufficient to render isotropic turbulence. This obviously affects the flow's kinetic energy spectrum. However, a standard compressible turbulence model may be applied. There is 
now increasing evidence showing that RM instabilities might be responsible for the emergence of turbulent flow in the reaction zone of unstable detonations $[\underline{16}, 19]$. Thus, compressible large-eddy simulation (LES) can be a proper tool to capture all the salient features of such detonation waves. The LES approach can be understood as a compromise between direct numerical simulations, in which all scales of the flowfield are resolved, and Reynolds averaged computations, which average over the entire spectrum of turbulence. In LES, the large energetic scales of turbulence are calculated explicitly and the subgrid scales (SGSs) are modeled.

In general, the simulation of detonations with resolved reaction zones is becoming progressively easier by the availability of adaptive mesh refinement (AMR) and high-performance computing. However, so far the effect of SGSs in reaction zones of unstable detonations has not been taken into account. In the case of highly unstable detonations, a clear need for SGS modeling has already been identified $[15,16,19]$. This work, therefore, aims at evaluating the role of SGSs in the reaction structure of an unstable detonation configuration. The turbulent reaction zone of an irregular detonation is studied using LES with the dynamic Smagorinsky turbulence model in combination with a simple Arrhenius kinetic reaction model. The role of hydrodynamic instabilities (i.e., RM and $\mathrm{KH}$ instabilities) in the consumption of unreacted gas pockets is examined. The results of LES are then compared with those obtained from solving Euler and Navier-Stokes (NS) equations for the same flow parameters. The influence of grid resolution on these solutions is then discussed.

The paper is organized as follows. The governing equations and the numerical method are presented in Sec. II. Results of the simulations are shown in Sec. III. The comparison with experiments is presented in Sec. IV. Three-dimensional (3-D) effects are discussed

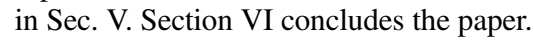

\section{Governing Equations and the Numerical Method}

Compressible flows are governed by the equations for conservation of mass, momentum, and energy; a transport equation for a reaction progress variable; and the thermodynamic equation of state. The two-dimensional (2-D) NS equations are expressed as follows:

$$
\frac{\partial U}{\partial t}+\frac{\partial F}{\partial x}+\frac{\partial G}{\partial y}+\frac{\partial F_{d}}{\partial x}+\frac{\partial G_{d}}{\partial y}=S
$$

where

$$
\begin{aligned}
U \equiv\left[\begin{array}{l}
\rho \\
\rho u \\
\rho v \\
\rho E \\
\rho \beta
\end{array}\right], \quad F \equiv\left[\begin{array}{l}
\rho u \\
\rho u^{2}+p \\
\rho u v \\
\rho u E+u p \\
\rho u \beta
\end{array}\right] \\
G \equiv\left[\begin{array}{l}
\rho v \\
\rho u v \\
\rho v^{2}+p \\
\rho v E+v p \\
\rho v \beta
\end{array}\right], \quad S \equiv\left[\begin{array}{l}
0 \\
0 \\
0 \\
0 \\
\rho W
\end{array}\right]
\end{aligned}
$$

and the diffusive fluxes are given by

$$
F_{d} \equiv\left[\begin{array}{l}
0 \\
-\tau_{x x} \\
-\tau_{x y} \\
-u \tau_{x x}-v \tau_{x y}+q_{x} \\
J_{x}
\end{array}\right], \quad G_{d} \equiv\left[\begin{array}{l}
0 \\
-\tau_{y x} \\
-\tau_{y y} \\
-u \tau_{y x}-v \tau_{y y}+q_{y} \\
J_{y}
\end{array}\right]
$$

The shear stresses, heat fluxes, and molecular fluxes are modeled by the usual molecular diffusion gradient mechanisms [23], yielding

$$
\begin{gathered}
\tau_{x x}=\mu\left(\frac{4}{3} \frac{\partial u}{\partial x}-\frac{2}{3} \frac{\partial v}{\partial y}\right), \\
\tau_{x y}=\tau_{y x}=\mu\left(\frac{\partial v}{\partial x}+\frac{\partial u}{\partial y}\right), \\
\tau_{y y}=\mu\left(\frac{4}{3} \frac{\partial v}{\partial y}-\frac{2}{3} \frac{\partial u}{\partial x}\right), \\
q_{x}=-K \frac{\partial T}{\partial x}, \quad q_{y}=-K \frac{\partial T}{\partial y} \\
J_{x}=-D \rho \frac{\partial \beta}{\partial x}, \quad J_{y}=-D \rho \frac{\partial \beta}{\partial y}
\end{gathered}
$$

Here, $S$ is the source term due to combustion. In these equations, $\rho, u$, $v, T$, and $p$ are, respectively, density, particle velocities in $x$ and $y$ directions of a Cartesian coordinate system, absolute temperature, and pressure. Further, $\tau, \mu, K$, and $D$ are, respectively, the stress tensor, dynamic viscosity, heat conductivity, and coefficient of mass diffusion. $\beta$ is the reaction progress parameter, which varies between one (for the unburned reactant) and zero (for the product). $E$ is the total energy per unit mass, which is defined as

$$
E=\frac{p}{\rho(\gamma-1)}+\frac{\left(u^{2}+v^{2}\right)}{2}+\beta Q
$$

in which $Q$ is the heat release per unit mass of the reactant and $\gamma$ is the ratio of the specific heats. $W$ in Eq. (2a) is the reaction rate, which follows the Arrhenius law as follows:

$$
W=-k \beta \exp \left(\frac{-E_{a}}{R T}\right)
$$

The perfect gas law is expressed as

$$
p=\rho R T
$$

where $R$ is a suitable gas constant for the present mixture taken as a suitable average value between the reacted and unreacted state. Temperature dependence for the kinematic viscosity, mass diffusion, and heat conduction is

$$
\frac{\mu}{\mu_{0}}=\left(\frac{T}{T_{0}}\right)^{m}, \quad \frac{K}{K_{0}}=\left(\frac{T}{T_{0}}\right)^{m}, \quad \frac{D}{D_{0}}=\left(\frac{T}{T_{0}}\right)^{m}
$$

in which $m=0.7$ is applied for a typical temperature dependence of these coefficients in reactive hydrocarbon flows [24].

The dependent variables are nondimensionalized with respect to the unburned mixture properties. The characteristic length scale used is the length travelled by a fluid particle from the leading shock to the position where $\beta=0.5$ in a Zeldovich, Doering, and von Neumann (ZND) structure. This is usually regarded as the half-reaction length (HRL). The dimensionless parameters are

$$
\begin{gathered}
p^{*} \frac{p}{\gamma p_{0}}, \quad \rho^{*} \frac{\rho}{\rho_{0}}, \quad T^{*}=\frac{T}{\gamma T_{0}}, \quad u^{*}=\frac{u}{c_{0}} \\
v^{*}=\frac{v}{c_{0}}, \quad c_{0}=\sqrt{\frac{\gamma p_{0}}{\rho_{0}}}, \quad E^{*}=\frac{E}{c_{0}^{2}}, \quad Q^{*}=\frac{Q}{R T_{0}} \\
E_{a}^{*}=\frac{E_{a}}{R T_{0}}, \quad W^{*}=\frac{l_{1 / 2}}{c_{0}} W, \quad \tau^{*}=\frac{\tau}{\gamma p_{0}}, \quad J^{*}=\frac{J}{\rho_{0} c_{0}} \\
x^{*}=\frac{x}{l_{1 / 2}}, \quad y^{*}=\frac{y}{l_{1 / 2}}, \quad t^{*}=\frac{c_{0}}{l_{1 / 2}} t \\
k^{*} \frac{l_{1 / 2}}{c_{0}} k, \quad \gamma=\frac{c_{p 0}}{c_{v 0}}
\end{gathered}
$$


In the following, for simplicity the " $*$ " is omitted. To implement LES, all the conservation equations are spatially filtered with a filter of size $\Delta$, which is generally equivalent to the grid size (hereafter called the "grid filter") of the LES simulation. In general, the filtered flow variable $f$ is denoted by an overbar as

$$
\bar{f}(x)=\int f\left(x^{\prime}\right) F\left(x-x^{\prime}\right) \mathrm{d} x^{\prime}
$$

where the integral is over the entire flow domain and $F$ is some filtering function. Here, we use a box filter for the spatial filter with the filter width $\Delta$ written in terms of the cell dimensions, i.e., $\Delta=\left(\Delta_{x} \Delta_{y}\right)^{1 / 2}$ [25-27]. These equations are subsequently Favre averaged, yielding

$$
\begin{aligned}
& U \equiv\left[\begin{array}{l}
\bar{\rho} \\
\bar{\rho} \tilde{u} \\
\bar{\rho} \tilde{v} \\
\bar{\rho} \tilde{E} \\
\bar{\rho} \tilde{\beta}
\end{array}\right], \quad F \equiv\left[\begin{array}{l}
\bar{\rho} \tilde{u} \\
\bar{\rho} \tilde{u}^{2}+\bar{p} \\
\bar{\rho} \tilde{u} \tilde{v} \\
\bar{\rho} \tilde{u} \tilde{E}+\tilde{u} \bar{p} \\
\bar{\rho} \tilde{u} \tilde{\beta}
\end{array}\right] \\
& G \equiv\left[\begin{array}{l}
\bar{\rho} \tilde{v} \\
\bar{\rho} \tilde{u} \tilde{v} \\
\bar{\rho} \tilde{v}^{2}+\bar{p} \\
\bar{\rho} \tilde{v} \tilde{E}+\tilde{v} \bar{p} \\
\bar{\rho} \tilde{v} \tilde{\beta}
\end{array}\right], \quad S \equiv\left[\begin{array}{l}
0 \\
0 \\
0 \\
0 \\
\bar{\rho} \bar{W}
\end{array}\right] \\
& F_{d} \equiv\left[\begin{array}{l}
0 \\
-\bar{\tau}_{x x}+\tau_{x x}^{\mathrm{SGS}} \\
-\bar{\tau}_{y x}+\tau_{y x}^{\mathrm{SGS}} \\
-\tilde{u} \bar{\tau}_{x x}-\tilde{v} \bar{\tau}_{x y}+\bar{q}_{x}+q_{x}^{\mathrm{SGS}} \\
\bar{J}_{x}+J_{x}^{\mathrm{SGS}}
\end{array}\right] \\
& G_{d} \equiv\left[\begin{array}{l}
0 \\
-\bar{\tau}_{x y}+\tau_{x y}^{\mathrm{SGS}} \\
-\bar{\tau}_{y y}+\tau_{y y}^{\mathrm{SGS}} \\
-\tilde{u} \bar{\tau}_{y x}-\tilde{v} \bar{\tau}_{y y}+\bar{q}_{y}+q_{y}^{\mathrm{SGS}} \\
\bar{J}_{y}+J_{y}^{\mathrm{SGS}}
\end{array}\right] \\
& \bar{\tau}_{x x}=\bar{\mu}\left(\frac{4}{3} \frac{\partial \tilde{u}}{\partial x}-\frac{2}{3} \frac{\partial \tilde{v}}{\partial y}\right) \quad \bar{\tau}_{x y}=\bar{\tau}_{y x}=\bar{\mu}\left(\frac{\partial \tilde{v}}{\partial x}+\frac{\partial \tilde{u}}{\partial y}\right) \\
& \bar{\tau}_{y y}=\bar{\mu}\left(\frac{4}{3} \frac{\partial \tilde{v}}{\partial y}-\frac{2}{3} \frac{\partial \tilde{u}}{\partial x}\right) \\
& \bar{q}_{x}=-\bar{K}^{-} \frac{\partial T}{\partial x}=-\bar{K} \frac{\partial \tilde{T}}{\partial x}, \quad \bar{q}_{y}=\overline{-K \frac{\partial T}{\partial x}}=-\bar{K} \frac{\partial \tilde{T}}{\partial y} \\
& \bar{J}_{x}=\overline{-D \rho \frac{\partial \beta}{\partial x}} \approx-\bar{D} \bar{\rho} \frac{\partial \tilde{\beta}}{\partial x}, \quad \bar{J}_{y}=\overline{-D \rho \frac{\partial \beta}{\partial y}} \approx-\bar{D} \bar{\rho} \frac{\partial \tilde{\beta}}{\partial y}
\end{aligned}
$$

The SGS stress tensor, $\tau_{i j}^{\mathrm{SGS}}=\bar{\rho}\left(\widetilde{u_{i} u_{j}}-\tilde{u}_{i} \tilde{u}_{j}\right)$ is modeled through the Smagorinsky eddy viscosity model [25]:

$$
\begin{gathered}
\tau_{i j}^{\mathrm{SGS}}-\frac{1}{3} \delta_{i j} \tau_{k k}^{\mathrm{SGS}}=-2 \bar{\mu}_{\mathrm{SGS}}\left(\tilde{S}_{i j}-\frac{1}{3} \delta_{i j} \tilde{S}_{k k}\right), \\
\tilde{S}_{i j}=\frac{1}{2}\left(\frac{\partial \tilde{u}_{i}}{\partial x_{j}}+\frac{\partial \tilde{u}_{j}}{\partial x_{i}}\right)
\end{gathered}
$$

Here, the eddy viscosity $\bar{\mu}_{\mathrm{SGS}}$ is a function of the filter size and the strain rate:

$$
\bar{\mu}_{\mathrm{SGS}}=\bar{\rho} C \bar{\Delta}^{2}|\tilde{S}|, \quad|\tilde{S}|=\sqrt{2 \tilde{S}_{i j} \tilde{S}_{i j}}
$$

In the classical model, $C$ is specified a priori and is often written as the Smagorinsky coefficient $\sqrt{ } C_{s}=C$ [27]. The isotropic part of the SGS stress tensor, $\tau_{k k}$, is modeled using the relation of Yoshizawa [28]:

$$
\tau_{k k}^{\mathrm{SGS}}=2 \bar{\rho} C_{l} \bar{\Delta}^{2}|\tilde{S}|^{2}
$$

Here, the model coefficient $C_{l}$ is expected to be of order 0.01 [29]. A major problem of the classical model is that the model coefficient $C$ [see Eq. (13)] is in reality dependent on the local flow conditions. To overcome this problem, Germano et al. [30] developed a procedure to dynamically calculate the model coefficient using local instantaneous flow conditions. This is written as

$$
\begin{aligned}
C \bar{\Delta}^{2} & =-\frac{L_{i j} M_{i j}-1 / 3 L_{i j} M_{m m}}{2\left(M_{i j} M_{i j}-1 / 3 M_{l l} M_{m m}\right)}, \\
L_{i j} & =\bar{\rho} \widehat{\tilde{u_{i}}} \tilde{u}_{j}-1 / \hat{\bar{\rho}}\left(\widehat{\bar{\rho} \tilde{u_{i}}}\right)\left(\widehat{\bar{\rho} \tilde{u_{j}}}\right), \quad M_{i j}=\alpha^{2} \hat{\bar{\rho}}|\hat{\tilde{S}}| \hat{\tilde{S}}_{i j}-\bar{\rho} \mid \widehat{\tilde{S} \mid \tilde{S}_{i j}}, \\
\alpha & =\hat{\bar{\Delta}} / \bar{\Delta}
\end{aligned}
$$

The SGS heat flux $q_{j}^{\mathrm{SGS}}=\bar{\rho}\left(\widetilde{u_{j} E}-\tilde{u_{i}} \tilde{E}\right)$ and the SGS reaction progress variable flux are, respectively, approximated as

$$
\begin{gathered}
J_{j}^{\mathrm{SGS}}=\bar{\rho}\left(\widetilde{u_{j} \beta}-\tilde{u_{i}} \tilde{\beta}\right)=-\frac{\bar{\mu}_{\mathrm{SGS}}}{S c_{t}} \frac{\partial \tilde{\beta}}{\partial x_{j}} \\
q_{j}^{\mathrm{SGS}}=\bar{\rho}\left(\widetilde{u_{j} E}-\tilde{u_{i}} \tilde{E}\right)=-\frac{\bar{\mu}_{\mathrm{SGS}}}{P r_{t}} \frac{\partial \tilde{E}}{\partial x_{j}}
\end{gathered}
$$

where $S c_{t}$ and $P r_{t}$ are turbulent Schmidt and turbulent Prandtl numbers, which are set to $P r_{t}=S c_{t}=0.9$. Finally, the Favre filtered equation of state is

$$
\bar{p}=\bar{\rho} R \tilde{T}
$$

\section{A. Chemical Kinetics Model}

The objective of this section is to describe a simple model for the LES filtered reaction rate $\bar{W}$ applicable to detonation waves. A detonation is a supersonic compression wave that moves into the fresh mixture and initiates the ignition of the unburned gases. After passage of the wave front, the pressure and temperature of the unreacted materials increase to an autoignition condition. The energy released by the subsequent combustion of the gas supports the shock front and preserves the self-sustained propagation of the detonation wave. To comply with these physics, the model of chemical kinetics should be compatible with a flow with significantly varying pressure. Further, it should capture the shock ignition mechanism and shockshear-layer interactions [31]. These set strong conditions for the applicable kinetic models and rule out many of those commonly used in other branches of turbulent reactive flows. This difficulty has been highlighted by Shepherd [19] and Dauptain et al. [31].

The Arrhenius law, which relates the chemical reaction rate to temperature variation, is widely used as the simplest model to simulate detonation waves (see, for example, $[1,15,16,22,32])$. As the reaction rate is highly nonlinear, the averaged reaction rate $\tilde{W}$ cannot be easily expressed as a function of the filtered variables, i.e., $\tilde{W} \neq W(\bar{\rho}, \tilde{T}, \tilde{\beta})[25]$. For this reason, reaction rate closures in turbulent combustion are usually derived from physical analysis, e.g., flame surface density (FSD) and $G$-equation-based combustion 
models. These models are functions of flame speed, burning velocity, flame geometry, and other properties [25]. However, due to the strong dependence of the reaction rates on the temperature variations due to lead shock compression, characteristic for detonations, such models cannot be used. In fact, because of the varying nature of turbulence in different combustion phenomena, application of a global model for chemical kinetics is extremely difficult.

In general, simulation of turbulent reactive flow in detonations involves two main issues. First, the shock front is the primary mechanism for reaction and ignition of the unburned gases [1]. Whereas turbulent mixing plays no role in the ignition mechanism at the vicinity of the shock front, the turbulence model should nevertheless be capable of considering the temperature variations due to shock compression. The Arrhenius law features this important capability, and the filtered reaction rate is then

$$
\tilde{W}=k \tilde{\beta}\left(\frac{-E_{a}}{R \tilde{T}}\right)
$$

This model assumes perfect mixing at the SGS level and neglects SGS fluctuations. The expression implicitly assumes that the turbulent subgrid time scale is shorter than all chemical time scales.

Second, previous investigations $[\underline{4}, \underline{12}, \underline{15}, \underline{22}]$ have shown that in unstable detonations most portions of the reactants cannot be ignited by shock compression and remain as unreacted gas pockets behind the main shock. These unreacted gases eventually burn due to diffusive turbulent mixing at pocket boundaries $[15,16]$. Thus, turbulent mixing may control the reaction rate in the reaction zone of unstable detonations. Another model is therefore required for turbulent simulation of the reaction rate behind the main shock. Moreover, transverse waves interact with these turbulent mixing regions, which results in the generation of a tremendous amount of vorticity [4]. In stable detonations, with a laminar reaction zone, no noticeable unburned pocket is observed behind the main shock and the classical shock compression still dominates the ignition mechanism. Thus, in stable detonations there is no need to use turbulence modeling in order to predict the laminar reaction zone of such detonations. Nevertheless, determination of proper turbulence models for turbulent detonations, which is the final state of the deflagration to detonation transition (DDT) phenomenon [24], remains an unresolved problem $[16,24]$. This is due to the unusual type of turbulence generated in this system by repeated shock-shearlayer interactions. The major mechanism of turbulence in gaseous detonations is a part of the DDT phenomenon[24] and includes RM instabilities induced by shock-flame interaction [4,16]. The interaction between the moving transverse wave and the turbulent shear layers leads to high levels of vorticity generation by RM instability [4]. On the contact surface separating the burned and unburned gases, this results in the simultaneous vorticity production in a range of scales extending from the smallest scale of the flame thickness to the detonation cell size. In addition to this, another source of turbulence in small scales is KH instabilities [16]. Hence, a large amount of vorticity is produced in the reaction zone of gaseous detonations. This turbulent flow is controlled by repeated transversewave-shear-layer interactions occurring at all scales. It is therefore markedly different than classical turbulence, which features isotropic, homogeneous, and equilibrium spectrum characteristics [24]. Characterization of this non-Kolmogorov turbulence and determination of the proper turbulent model demand further research [24].

The present work puts forward an attempt at SGS modeling through the simple model of Eq. (19) based on the filtered quantities. This is the simplest model compatible with the physics of the problem. It should be noted that, in the course of the present study, other subgrid chemical models, such as those based on the FSD and a flamelet concept [26], were tried. None of the resultant flowfields supported a self-sustained detonation wave. This is because these models do not include the sensitivity of the heat release to the temperature rise by shock compression. Although there has been extensive research on more sophisticated LES models for compressible flows tailored to classical isotropic turbulence [33], the non-Kolmogorov type turbulence in detonation waves [7] and DDT [24] complicates the choice of a suitable SGS model for detonations. For example, because the Smagorinsky model does not disappear in regions where all dynamic scales are resolved, the SGS viscosity needs to be modified [33]. It seems that the mixed scale model is also a proper model. This model considers the SGS viscosity as a combination of the second invariant of the stress tensor of the characteristic length and of the kinetic energy of the smallest resolution [33]. This is an ongoing research by the authors to verify the suitability of different models for accurate predictions of detonation propagation.

\section{B. Numerical Method}

The governing equations are solved by the fractional step method [34-36]. The convective flux, diffusive, and source terms are integrated separately during successive substeps within each global time step. First, the convective terms are addressed by solving

$$
\frac{\partial \tilde{U}}{\partial \tilde{t}}+\frac{\partial \tilde{F}}{\partial \tilde{x}}+\frac{\partial \tilde{G}}{\partial \tilde{y}}=0
$$

Equation (20) represents the 2-D homogeneous Euler equations of gas dynamics in Cartesian coordinates. The system of Eq. (20) is discretized with the unsplit upwind method of Colella [37] and Saltzman [38] as follows:

$$
\begin{aligned}
\tilde{U}_{i, j}^{n+1}= & \tilde{U}_{i, j}^{n}+\frac{\Delta \tilde{t}}{\Delta \tilde{x}}\left[F\left(\tilde{U}_{i-1 / 2, j}^{n+1 / 2}\right)-F\left(\tilde{U}_{i+1 / 2, j}^{n+1 / 2}\right)\right] \\
& +\frac{\Delta \tilde{t}}{\Delta \tilde{y}}\left[G\left(\tilde{U}_{i, j-1 / 2}^{n+1 / 2}\right)-G\left(\tilde{U}_{i, j+1 / 2}^{n+1 / 2}\right)\right]
\end{aligned}
$$

where

$$
\tilde{U}_{i, j}^{n}=\int_{\Delta_{i, j}} \tilde{U}\left(\tilde{x}, \tilde{y}, \tilde{t}^{n}\right) \mathrm{d} \tilde{x} \mathrm{~d} \tilde{y}
$$

$F\left(\tilde{U}_{i+1 / 2, j}^{n+1 / 2}\right)$ and $G\left(\tilde{U}_{i, j+1 / 2}^{n+1 / 2}\right)$ are the time averaged approximate fluxes at the cell interfaces. The state variable vectors $\tilde{U}_{i+1 / 2, j}^{n+1 / 2}$, $\tilde{U}_{i, j+1 / 2}^{n+1 / 2}, \tilde{U}_{i, j-1 / 2}^{n+1 / 2}$, and $\tilde{U}_{i-1 / 2, j}^{n+1 / 2}$ are the time averaged conservative variables at the cell interfaces. These variables are determined as the solution of the Riemann problem projected in the $x$ and $y$ directions with the left and the right states $\left(\tilde{U}_{i+1 / 2, j, L}^{n+1 / 2}, \tilde{U}_{i+1 / 2, j, R}^{n+1 / 2}\right)$ and $\left(\tilde{U}_{i, j+1 / 2, D}^{n+1 / 2}, \tilde{U}_{i+1 / 2, j+1 / 2, U}^{n+1 / 2}\right)$. The solution of Eq. (20) is used as the initial condition for the subsequent fractional substep. This aims at incorporating the effect of the diffusive and SGS terms in the governing equations [Eq. (1)]. The diffusive step takes the form of

$$
\frac{\partial \tilde{U}}{\partial \tilde{t}}=\frac{\partial \tilde{F}_{d}}{\partial \tilde{x}}+\frac{\partial \tilde{G}_{d}}{\partial \tilde{y}}
$$

To solve the preceding equation, the diffusive terms are discretized by second-order central differences to model spatial gradients.

Finally, the last substep, involving the reactive source terms, uses the solution of Eq. (23) as the initial condition to solve a system of ordinary differential equations given by

$$
\frac{\partial \tilde{U}}{\partial \tilde{t}}=\tilde{S}
$$

The final splitting algorithm is

$$
\tilde{U}^{n+1}=L_{H}^{\Delta t} L_{D}^{\Delta t} L_{C}^{\Delta t} \tilde{U}^{n}
$$

in which $L_{H}^{\Delta t}$ represents the solution of the hyperbolic Eq. (20), $L_{D}^{\Delta t}$ is the solution of the diffusive Eq. (23), and $L_{C}^{\Delta t}$ stands for the solution of Eq. (24).

Because of the presence of intense reactions near the shock front, it is necessary to use very fine meshes in this region. To fulfil this 
requirement, a simple version of the AMR of Berger and Colella [39] was used in the present work. Two sets of uniform grids have been used to discretize the computational domain. The entire domain was covered by coarse grids, and fine meshes were superimposed on the coarse grids in the vicinity of the front. Fine grid boundaries were always coinciding with coarse grid faces. This method has been used by Bourlioux [40] in one-dimensional (1-D) and 2-D numerical simulations and Mazaheri [41] in 1-D numerical simulation of detonation waves. The global procedures of 1-D and 2-D AMR are similar. The solution at those coarse cells adjacent to the fine grids is corrected in order to preserve conservation [41].

\section{Boundary and Initial Conditions and Grid Resolution}

The detonation runs from left to right in the positive $x$ direction in a 2-D channel. Because the fluid ahead of the detonation is in its quiescent state, the right-hand boundary condition is irrelevant. The boundary conditions imposed on the lower and the upper sides of the channel are reflecting or free-slip solid wall boundary conditions.

To reduce the computational time, a nonreflecting boundary condition (NRBC) is imposed on the left side of the domain. This boundary condition is similar to those used as convective boundary conditions in incompressible flow to prevent reflection of pressure waves at the boundary [26]. The details of this boundary condition can be found elsewhere [16,42]. The existence of an average Chapman and Jouguet state, at the end of reaction zone, makes this concept attractive for simulating detonation waves [43]. Considering this concept, the NRBC is used to truncate the computational domain. The problem of determining a computational domain width suitable to investigate periodic detonation structures is a nontrivial one. Using a 1-D simulation of a pulsating overdriven detonation wave in a mixture with $E_{a} / R T_{0}=50, Q / R T_{0}=50$, and $\gamma=1.2$, Hwang et al. [44] reported the minimum domain length of $L=75$ HRL. This was required to keep the flow properties unaffected by the truncation of the computational domain. Kasimov and Stewart [45] used a domain length of $L=20 \mathrm{HRL}$ for mixtures with $Q / R T_{0}=50, \gamma=1.2$, and activation energies from 25 to 28 , and they used $L=60 \mathrm{HRL}$ for an activation energy of 35. Han [42] used NRBC in 1-D and 2-D simulations of a detonation wave, for a mixture with $E_{a} / R T_{o}=10, Q / R T_{o}=50, \gamma=1.2$, and $f=1.0$. He concluded that the domain length should be over 13.8 HRL. These studies showed that the minimum domain length depends on the activation energy. As $E_{a} / R T_{o}$ decreases, the required minimum domain length also decreases. Further, the effect of domain length on the detonation structure was examined by the present authors in a separate work (unpublished). Both 1-D and 2-D simulations were conducted to determine the minimum domain length that is required for the present NRBC. The solution of the whole domain was compared with the truncated solution domain for different activation energies. It was found that as the activation energy increases from 10 to 27.4 the minimum acceptable domain length varies from $20 \mathrm{HRL}$ to $60 \mathrm{HRL}$. Therefore, in this work $L \cong 50 \mathrm{HRL}$ was chosen for a mixture with $E_{a} / R T_{o}=20$.

Table 1 shows the thermophysical properties of the mixture considered in the present work also used in other investigations (e.g.,

Table 1 Thermophysical properties of the mixture

\begin{tabular}{lc}
\hline \hline Parameter & Value \\
\hline$p_{0}$ (initial pressure) & $10^{5} \mathrm{~Pa}$ \\
$\rho_{0}$ (initial density) & $1.161 \mathrm{~kg} \cdot \mathrm{m}^{-3}$ \\
$T_{0}$ (initial temperature) & $300 \mathrm{~K}$ \\
$\gamma$ (adiabatic index) & 1.2 \\
$E_{a}$ (activation energy) & $11.94 \mathrm{kcal} \cdot \mathrm{mol}^{-1}$ \\
$E_{a} / R T_{o}$ (dimensionless activation energy) & 20 \\
$E_{a} / R T_{p s}$ & 3.6 \\
$Q$ (chemical energy released) & $29.79 \mathrm{kcal} \cdot \mathrm{mol}^{-1}$ \\
$Q / R T_{o}$ (dimensionless chemical energy & 50 \\
released) & \\
$K_{0}$ (conductivity coefficient) & $3.179 \times 10^{-2} \mathrm{~W} \cdot \mathrm{m}^{-1} \cdot \mathrm{K}^{-1}$ \\
$\mu_{0}($ viscosity) & $1.846 \times 10^{-5} \mathrm{~N} \cdot \mathrm{s} \cdot \mathrm{m}^{-2}$ \\
$D_{0}$ (mass diffusion coefficient) & $1.59 \times 10^{-5} \mathrm{~m}^{2} \cdot \mathrm{s}^{-1}$ \\
\hline \hline
\end{tabular}

$[16,46,47])$. Detonation structures in this mixture have been extensively studied both numerically $[10,12,15,22,32,48-53]$ and experimentally $[15,54-56]$. The activation energy corresponds to the mixtures such as hydrogen-oxygen, acetylene-oxygen, and methane-oxygen at low pressures (see [7,10,15,54,57-59]).

Further, recent numerical studies of detonations have shown that grid resolution can have a profound effect upon the simulated flow [12]. The numerical simulation of Sharpe [32] showed that the detonation in a mixture with $E_{a} / R T_{o}=20$ exhibits regular structure. Mahmoudi and Mazaheri [12], however, argued that the regularity of detonation structure depends on the grid resolution. They demonstrated that for resolutions higher than 125 cells per HRL the structure is irregular with a highly turbulent reaction zone. However, for low grid resolution the structure is regular with a laminar reaction zone.

In nondimensional terms, the system is characterized such that Reynolds, Schmidt, Prandtl, and Lewis numbers are $R e=\frac{\rho_{0} c_{0} l_{1 / 2}}{\mu} \sim 10^{4}, P r=\frac{\mu c_{p_{0}}}{K}=1, S c=\frac{\mu}{\rho_{0} D}=1$ and $L e=\frac{S c}{P r}=1$.

To initiate the detonation, a strong blast wave is located close to the left boundary, which moves to the right and forms a 1-D detonation. For a strong blast wave with the shock Mach number $M_{s}$ at the location $R_{s}$ with respect to the center of initiation, the initiation energy is obtained from

$$
\frac{E_{0}}{p_{0}} \cong R_{s} \gamma M_{s}^{2} I
$$

as suggested by Mazaheri [41]. In this equation, $I$ is a function of only $\gamma$ and $p_{0}$ is the pressure of the fresh mixture. To initiate a detonation, a large amount of energy (higher than the critical energy) is deposited instantly in the mixture. In the present work with $\gamma=1.2$ the value of $I$ is found to be 2.622. The 1-D detonation is then perturbed by adding a disturbance in a fresh mixture density between $R_{s}$ and $R_{s}+1$ $[\underline{8}, \underline{32}, \underline{41}, \underline{53}]$. This disturbance has the form

$$
\rho^{\prime}= \begin{cases}0 & x<R_{s} \\ 0.25[1+\cos (\pi y / w)] \sin \left(\pi\left(R_{s}+1-x\right)\right), & R_{s} \leq x \leq\left(R_{s}+1\right) \\ 0 & x>\left(R_{s}+1\right)\end{cases}
$$

where $w$ is the width of the domain. The perturbation quickly leads to the formation of transverse waves and a 2-D detonation in the channel.

It is well demonstrated that to correctly resolve the time-dependent structure of the detonations by numerical simulations very fine meshes should be used $([12,32,53,60])$. In a 2-D simulation, Sharpe [32] observed that a grid resolution less than about 20 cells in HRL gives poor prediction of the detonation structure. $\mathrm{Hu}$ et al. [49] conducted 2-D simulations with a resolution of about 440 cells in the reaction zone length of a ZND detonation. These authors [34] note that a sufficiently high resolution and a complex detailed chemical reaction model are both needed to resolve the detonation structure. Furthermore, Mahmoudi and Mazaheri [12] reported for a mixture with moderate activation energy $\left(E_{a} / R T_{o}=20, Q / R T_{o}=50\right.$, and $\gamma=1.2)$ that at least 125 cells per HRL are required to correctly capture the hydrodynamic instabilities in irregular structure detonations. However, in mixtures with lower activation energy (mixtures with $E_{a} / R T_{o}=10, Q / R T_{o}=50$, and $\gamma=1.2$ ), a total of 25 cells per HRL reveals a good solution of regular detonations. Thus, in the present work, for a mixture with $E_{a} / R T_{o}=20$, a resolution of 600 points in HRL is employed.

A scalable parallel code was developed to carry out the timeconsuming 2-D computations. All calculations were performed on a parallel machine with 12 nodes in which each node has two CPUs and each CPU contains six cores. The processors were Intel ${ }^{\circledR}$ Xeon ${ }^{\circledR}$ X5167 with a clock speed of $2.93 \mathrm{GHz}$ and up to $288 \mathrm{~GB}$ of memory. The parallelization is based on the distributed-memory principle, in which the computational domain is divided into different computational zones and spread among the different nodes. The message passing interface communication library was chosen for 
parallelizing the code. Using double precision accuracy, the typical computation time for a resolution of 600 cells per HRL for channel width $(w=6)$ and using double precision accuracy took about three weeks to allow the detonation to run for $450 \mathrm{HRL}$.

\section{Results}

\section{A. Detonation Structure}

Figures $\underline{1 \mathrm{a}}$ and $\underline{1 \mathrm{~b}}$ visualize the contours of density of the detonation structure for the solution of Euler and NS equations, respectively, where primary triple point A moves downward. The upper part of triple point A is the Mach stem (M), and the lower part is the incident wave (I). Figure 1a represents the Euler structure, in which secondary triple points $\overline{\mathrm{B}}$ and $\mathrm{C}$ are marked. Further, in this figure, the vortical structure that gives rise to the appearance of an irregular structure is observed. The details of this structure were previously discussed by Mahmoudi and Mazaheri [12]. The interaction of triple point A with the bottom wall produces a region of high pressure and temperature. The high-pressure zone propagates in a pair of forward and backward facing jets. As the jets spread, they undergo an $\mathrm{RM}$ instability involving the baroclinic $\nabla p \times \nabla \rho$ vorticity production mechanism $[4,12,16,32]$. This is in agreement with the recent experimental observation of Bhattacharjee et al. [14]. They found that the jet flows significantly enhance the mixing behind the Mach stem and lead to the reaction of substantial amounts of unburned gases [14]. The forward jet impacts on the new Mach stem and causes the appearance of secondary triple point B $[12,22,61]$. Interaction of the large vortical structure along the primary shear layer with the shock front results in the formation of secondary triple point $\mathrm{C}$ close to the upper boundary $[12,22,61]$.

Figure $1 \mathrm{~b}$ shows the detonation structure obtained by solving the NS equations. It is observed that the results of Euler and NS simulations are qualitatively similar. In Figs. $1 \mathrm{a}$ and $1 \mathrm{~b}$, the secondary triple points and the vortical structures are clearly visible. However, the small scale vortices produced by KH instabilities are suppressed in the NS solution. Details of the difference between Euler and NS solutions are explained further by Mazaheri et al. [16]. In short, diffusion suppresses the small scale vortices produced by $\mathrm{KH}$ instabilities and decreases the turbulent mixing rate of burned and partly burned gases along shear layers. Figure $1 \mathrm{c}$ shows the structures obtained from LES. Clearly, LES reveals a much more regular structure compared with those obtained by NS and Euler simulations. Although the small scale KH vortices are seen in all simulations, the size of these vortices is much smaller in the LES solution. This is due to the high dissipation in LES, which suppresses the small scale hydrodynamic instabilities $[\underline{4}, \underline{12}, \underline{15}, \underline{16}]$.

Figure 1c also shows that the long tongue of unreacted gases associated with the jet flow has almost vanished in the LES structure.
Further, the jet flow at the lower wall is absent in Fig. 1c, and the unreacted gases entrained into the jet region are also more rapidly reacted. This faster burnout rate is particularly evident in the LES solution. Elimination of the jet flow results in cooler burned gas inside the rolling zone of the jet behind the incident wave. Consequently, the turbulent mixing decreases in this region. This leads to a longer reaction zone length behind the incident wave. Secondary triple point $\mathrm{B}$, which is visible in Figs. 1a and $1 \mathrm{~b}$, vanishes in Fig. 1c. The incident wave propagates as an oblique wave in the Euler and NS solutions. Nonetheless, it moves like a normal wave in the LES structure. Interaction of the jet with the shock front accelerates the lower part of the main shock with respect to the other parts and creates secondary triple point B. Because of the elimination of the jet flow in LES, such interactions cannot occur and therefore triple point B disappears. Further, due to consumption of the tongue pocket in LES, interaction of the transverse wave and tongue pocket does not occur. Consequently, the transverse wave takes a simpler structure as it passes behind the main shock in the LES structure.

Figures $2 \mathrm{a}-2 \mathrm{c}$ represent contour plots of the reaction progress variable within the detonation structure. It is seen that the size of the jet flow close to the lower and upper boundary in the NS simulation is lower than that of the Euler solution. Once again, in the LES solution the jet flow almost disappears. Moreover, the unreacted gases that accumulate in the rolling zone of the upper jet are consumed faster in Fig. 2c. Hence, a brighter region is observed inside the upper jet in the LES structure. Figure $2 \mathrm{c}$ also shows that the reaction zone length behind the incident wave in LES is $L_{1 / 2} \cong 0.45$, which is much longer than that of the Euler solution, i.e., $L_{1 / 2} \cong 0.09$. This is due to the elimination of the lower jet in the LES structure. Thus, the amount of hot gases behind the incident wave decreases. It follows that the gases passing through the incident wave cannot be reacted and the reaction zone length increases. On the other hand, the value of the reaction progress variable (ensemble averaged) in a certain section in a LES solution is $\beta \cong 0.91$, which is lower than that of the Euler and NS solutions, $\beta \cong 0.98$. It should be noted that the length of the reaction zone behind the incident wave increases in the LES solution. However, a high dissipation rate in the LES structure enhances the reaction rate and reduces the reaction progress variable.

Figures $3 \mathrm{a}-3 \mathrm{c}$ show the pressure contours of the detonation structure. Figure 3 a uncovers that the interaction of the strong portion of the main transverse wave (labeled M.T.W in the figure) with the unburned pocket produces a highly pressurized region with $p \approx 75$. A careful comparison between Figs. $3 \mathrm{a}$ and $3 \mathrm{c}$ reveals that the value of pressure in the highly pressurized region in the LES solution has decreased to $p \approx 45$. This is because of the fast consumption of unburned pockets in the LES structure, which cancels the interaction of the transverse wave with the unburned pocket. The strength of the strong portion of the transverse wave (O-A in Fig. 3 ) is similar in the

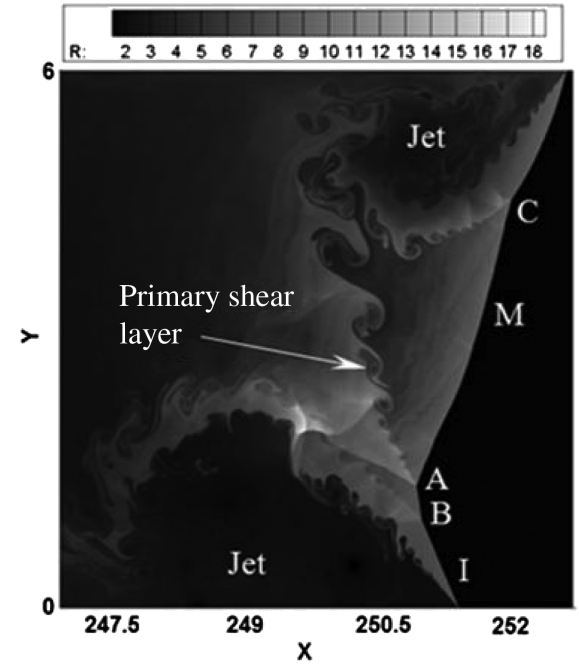

a) Euler

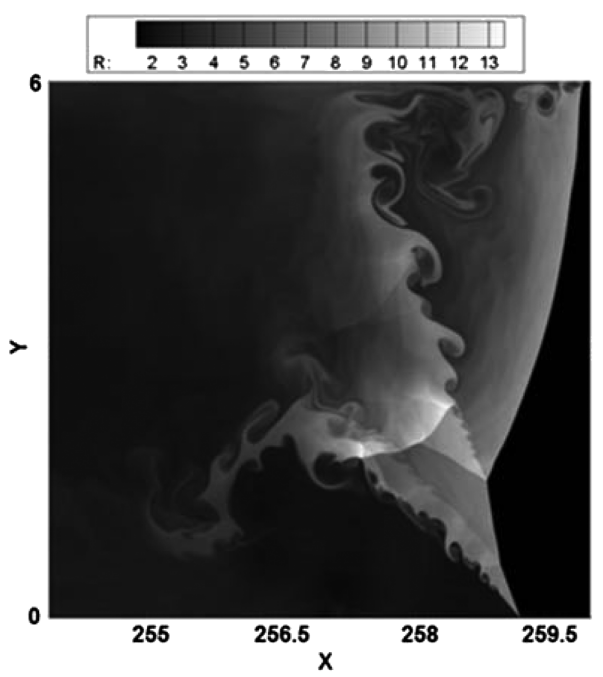

b) Navier-stokes

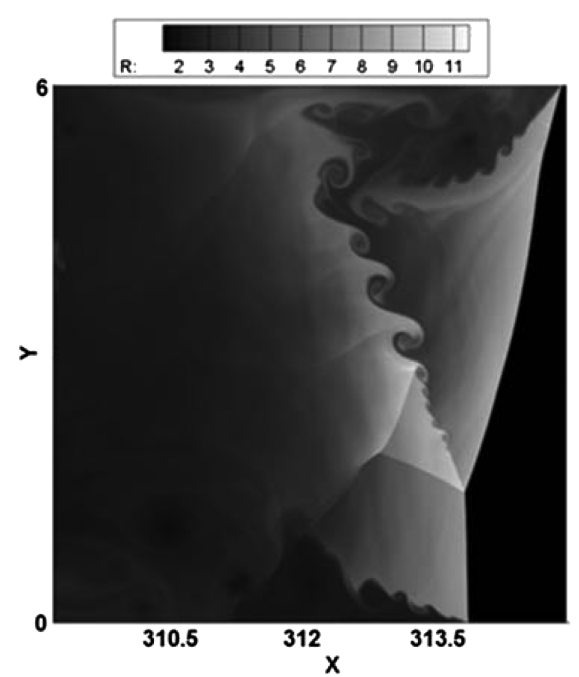

c) LES

Fig. 1 Contour of the density of the detonation structure in a mixture with $E_{a} / R T_{o}=20, Q / R T_{o}=50, \gamma=1.2$, and a resolution of 600 cells per $\mathrm{HRL}$. 


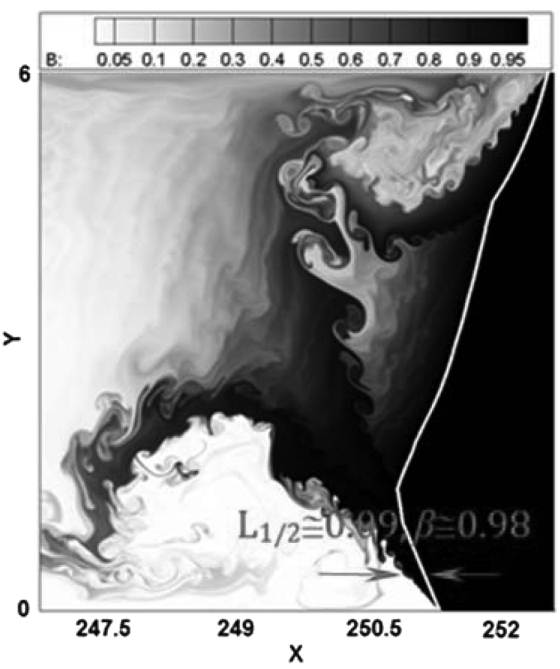

a) Euler

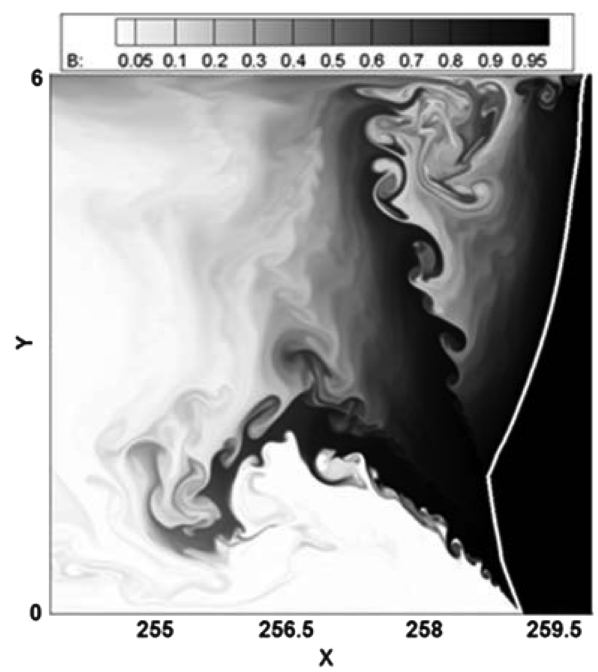

b) Navier-Stokes

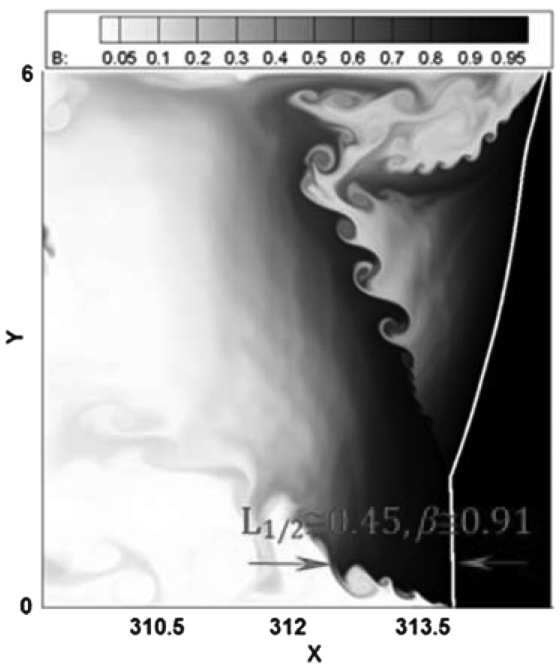

c) LES cells per HRL.

three simulations and is about $S \cong 0.8$. Here, strength is defined as $S=\left(P_{2}-P_{1}\right) / P_{1}$, where $P_{1}$ and $P_{2}$ are the pressure on each side of the wave. Interesting to note is that in the Euler and NS structures the rare section of the transverse waves does not interact with the bottom wall. However, in the LES solution the extended portion of the transverse wave collides with the wall and creates a reflective wave before the triple point reaches the wall (see Fig. $\underline{3 \mathrm{c}}$ ).

\section{B. Effect of Resolution}

To determine the role of grid resolution on the detonation structure obtained by LES, NS, and Euler simulation, the computations were repeated for a resolution of 250 cells per HRL. Figures $4 a-4 c$ show the outcome. Comparison of these figures reveals that in a low grid resolution situation the structures obtained by three simulations are qualitatively similar. The upper and lower jets and the long tongue pocket are clearly visible in the three figures.

Comparison of Figs. $4 \mathrm{a}$ and $4 \mathrm{~b}$ with Figs. $2 \mathrm{a}$ and $2 \mathrm{~b}$ indicates that as the resolution increases from 250 to $6 \overline{00}$ cells per HRL the detonation structures obtained by these resolutions are very similar. Therefore, within this range, Euler and NS solutions remain resolution independent. However, in the LES solution, as the resolution increases from 250 to 600 cells per HRL, the tongue pocket disappears in Fig. 4c. The incident wave propagates like a normal wave in the LES solution for a resolution of 250 cells per HRL.
Nonetheless, under a resolution of 600 cells per HRL, this wave moves like an oblique wave. This is due to the existence of a large jet flow close to the lower wall, which results in the acceleration of the lower portion of the main shock with respect to the other portions. It is expected that in NS computations (with a laminar diffusive coefficient) as the resolution increases the numerical diffusion decreases. Hence, the physical diffusion dominates the numerical diffusion. In the solution with 600 cells per HRL the physical diffusion exceeds the numerical diffusion, yet the total diffusion is not high enough to result in the consumption of the unburned pockets. In turn, the effect of dissipation in small scale vortices is well resolved with higher resolution, which results in higher burning of the unburned gas pockets. Thus, as the resolution increases, finer vortical features appear in the structure (see Figs. 2 and 4 ). This indicates that in addition to laminar diffusion turbulent diffusion should be taken into account in the numerical simulation of gaseous detonations using a proper turbulence model $[15,16]$. In the LES computations, increasing the resolution from 250 to 600 cells per HRL leads to the consumption of the tonguelike unburned pockets. This, once again, shows that by increasing the resolution the amount of diffusion (i.e., laminar diffusion and turbulent diffusion) exceeds the numerical diffusion. In contrast to Euler and NS solutions, the physical diffusion in LES is high enough to consume the unburned gases. In low grid resolution, however, the turbulent mixing produced by hydro-

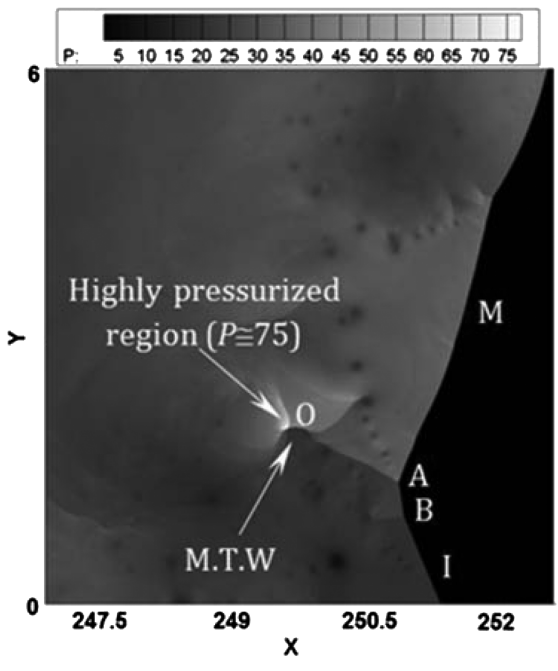

a) Euler

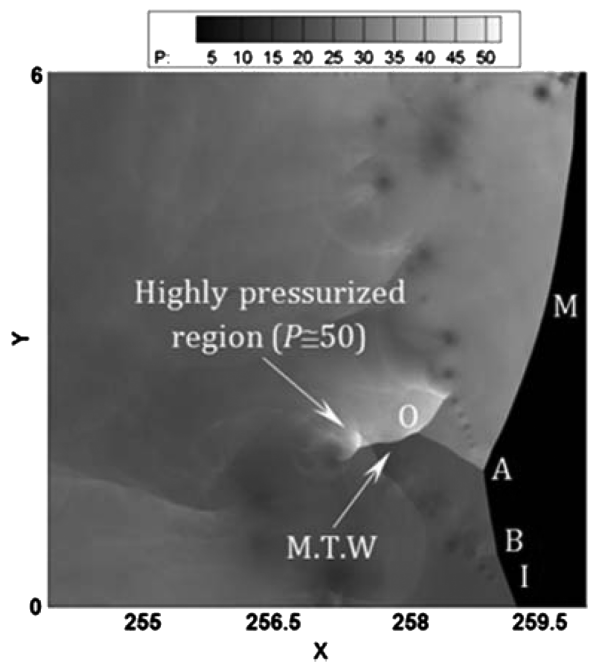

b) Navier-stokes

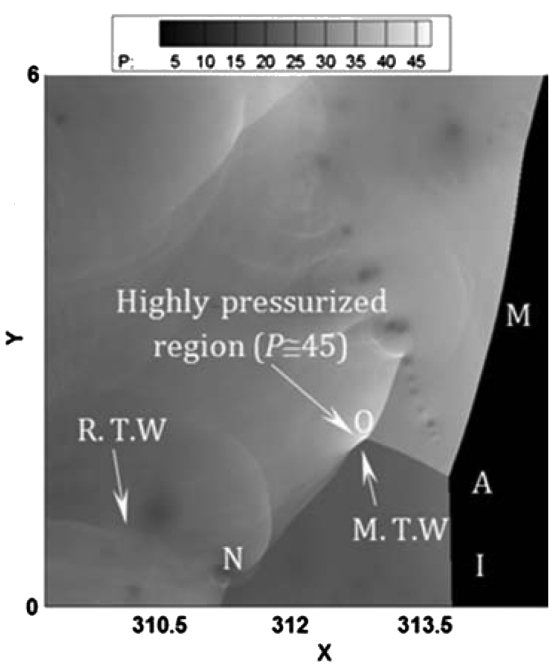

c) LES

Fig. 3 Pressure contour of the detonation structure in a mixture with $E_{a} / R T_{o}=20, Q / R T_{o}=50, \gamma=1.2$, and resolution of 600 cells per HRL. 


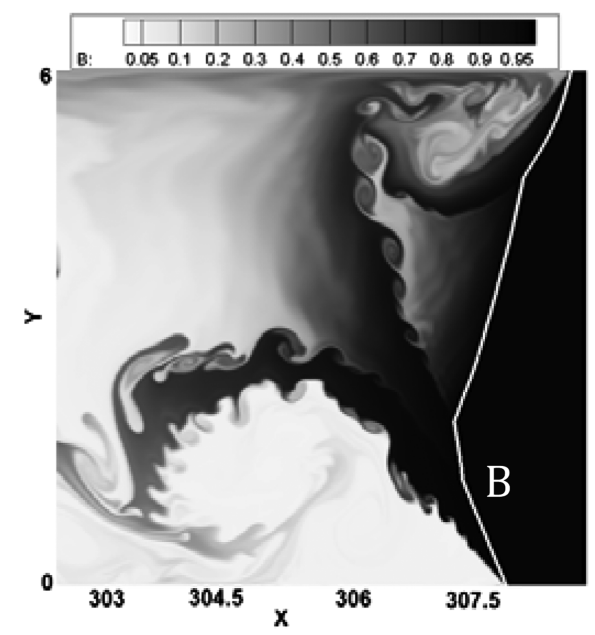

a) Euler

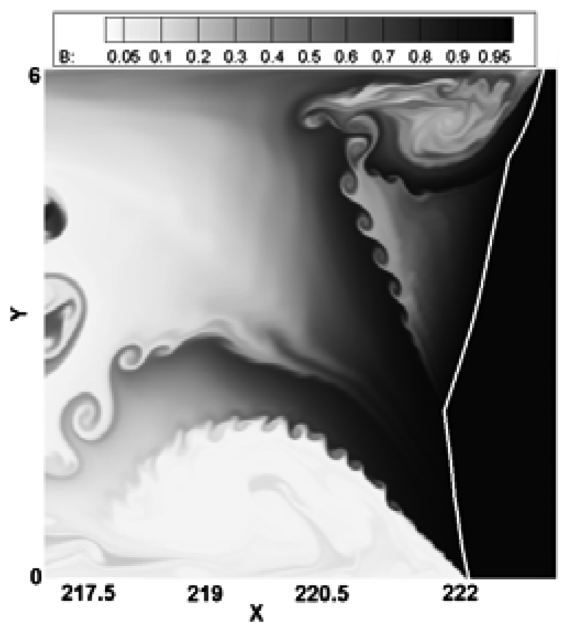

b) Navier-Stokes

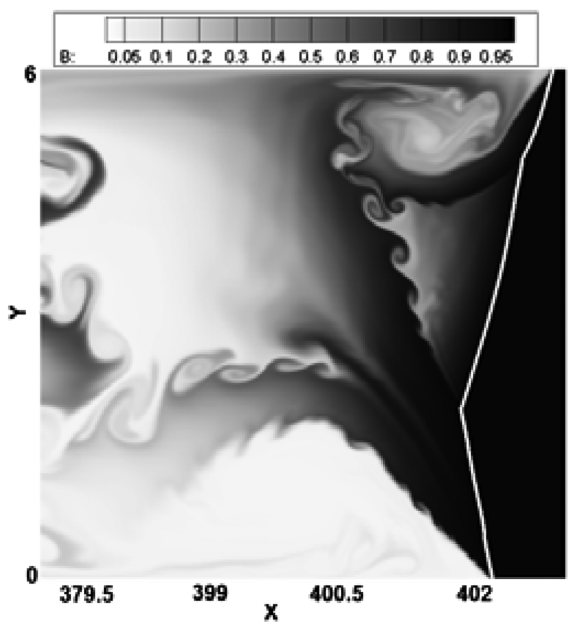

c) LES

Fig. 4 Contour of the reaction progress variable of the detonation structure in a mixture with $E_{a} / R T_{o}=20, Q / R T_{o}=50, \gamma=1.2$, and resolution of 250 cells per HRL.

dynamic instabilities (i.e., $\mathrm{KH}$ and $\mathrm{RM}$ instabilities) has a profound role in the consumption of unreacted gases. Therefore, for LES to result in a reasonable structure of gaseous detonations a resolution of 600 cells per HRL is required to capture the effects of dissipation at SGSs.

\section{Turbulent Viscosity}

The results presented in this section show that the dissipation at SGS is high enough to burn the tonguelike unreacted pocket. The question may arise how the dissipation introduced here by the LES model compares with the real turbulent diffusion. In LES, reacting flows are Favre filtered and turbulence closure is achieved using the dynamic Smagorinsky subgrid model. The Smagorinsky coefficient may thereby take positive or negative values. A positive value implies that energy flows from the resolved to the SGSs, which is in agreement with the cascade hypothesis, whereas a negative coefficient implies an inverse cascade or "backscatter." Figure 5 shows a snapshot indicating the dimensionless SGS viscosity in the reaction zone structure of detonation in a mixture with $E_{a} / R T_{0}=20$ and a resolution of 600 cell per HRL. In this figure, the turbulent viscosity takes a wide range from $-1.5 \times 10^{-4}$ to $4 \times 10^{-4}$. This is in accord with the real turbulent detonation, which has a wide range of viscosity in different regions of the reaction zone. The positive value of $\mu_{\mathrm{SGS}}$ corresponds to the region close to the transverse wave behind the main shock. The negative values are more evident in the rolling

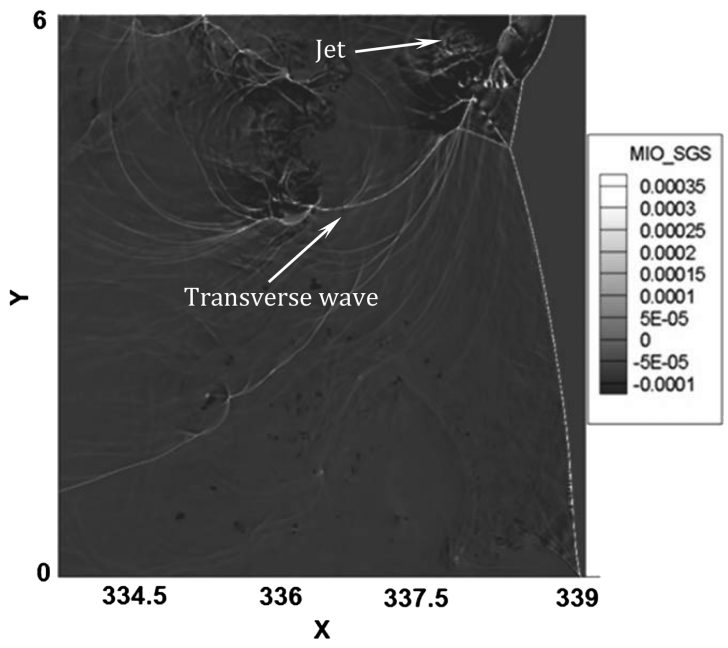

Fig. 5 Contour of dimensionless subgrid scale viscosity $\mu_{\mathrm{SGS}}$ behind the shock front in a mixture with $E_{a} / R T_{o}=20, Q / R T_{o}=50, \gamma=1.2$, and a resolution of 600 cells per HRL. zone of the jet flow close to the upper wall. In comparison to the laminar dimensionless viscosity for the NS case considered in the present work, which is about $10^{-4}$, one can conclude that the turbulent viscosity is up to an order of magnitude (i.e., approximately four times) larger than the laminar viscosity. This range of turbulent viscosity is in agreement with the measured real viscosity of Radulescu et al. [15]. They determined the kinematic viscosity in the burned products of a methane-oxygen mixture with the Gordon and McBride equilibrium code. They reported that the kinematic viscosity in their experiment is about $5 \times 10^{-4} \mathrm{~m}^{2} / \mathrm{s}$. Comparing with the laminar viscosity of about $10^{-4}$ adopted for the NS simulations, which is an order of magnitude lower than the viscosity in real systems, one can conclude that the viscosity introduced in LES for a resolution of 600 cells per HRL (i.e., $4 \times 10^{-4}$ ) is in the range of the viscosity in the real systems.

It is worthwhile to recall that the turbulence in a detonation is induced by repeated shock-shear-layer interactions. Because of such interactions, vorticity is generated on a range of scales simultaneously, from the scale of the system to the smallest scale of the laminar flame thickness. Without using a turbulence model, the effect of turbulent transport in this wide range of spectrum cannot be well resolved. Therefore, in order to determine the long-term behavior of the detonation structure, a compressible turbulence model (e.g., LES) should be implemented to take into account a broad range of turbulent viscosity values.

\section{Comparison of LES with Experiment}

To validate the LES results, this section compares the LES structures with the experimental observations of Kiyanda [62] and Bhattacharjee [13]. Figures $\underline{6 a}-\underline{6 c}$ show the schlieren images of Kiyanda [62] in an oxygen-methane mixture of initial pressure of $20 \mathrm{kPa}$. It should be noted though that the experimental images are for an oxygen-methane mixture, which features a higher activation energy than the mixture considered in the present simulation. The comparison with experiments is therefore qualitative and meant to show turbulent detonation in the same scale as in the simulations. We compare the tonguelike gas pockets in Euler, NS, and LES structures against the experimental observations to determine which one is capable of capturing realistic detonation structures.

In Fig. 6a, the triple point has reflected off the upper wall and moves downward into the incident wave. Figure $\underline{6}$ clearly shows the short reaction zone behind the strong Mach stem wave and a longer reaction zone behind the incident wave. Collision of the triple point with the wall results in the detachment of an unburned pocket from the wave front. Figures $6 \mathrm{a}-6 \mathrm{c}$ show that as the triple point moves downward the size of the unreacted gas pocket decreases. Both Radulescu [15] and Kiyanda [62] report that the unburned pockets 


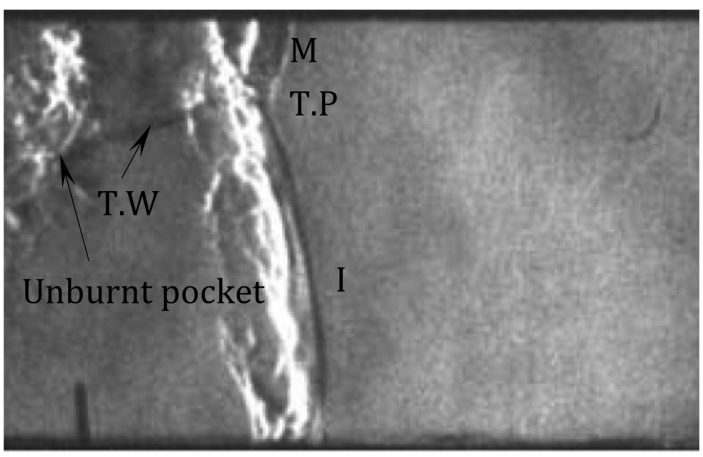

a)

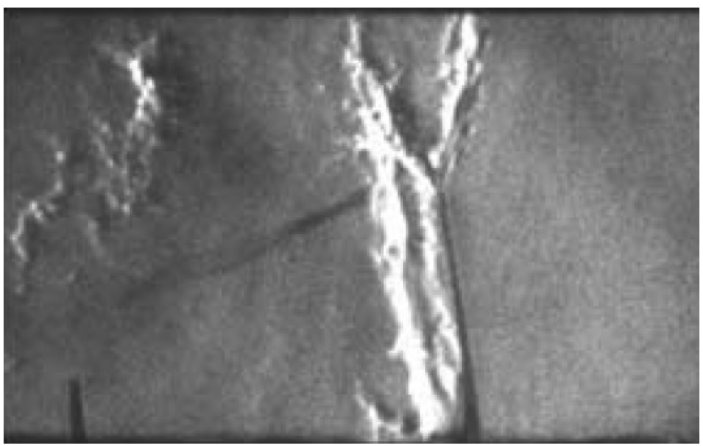

b)

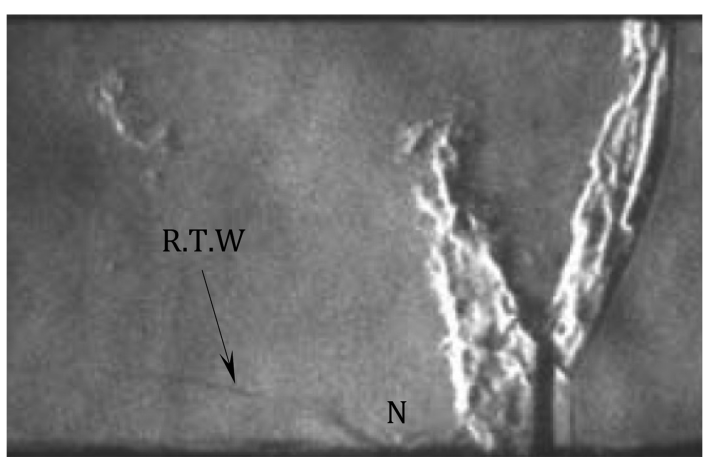

c)

14 Fig. 6 Schlieren of the reaction zone structure of the detonation [62].

continue to remain behind the main shock for four to six halfreaction-zone lengths. It is concluded that the diffusion of hot and reacted neighboring gases to the unburned pockets results in fast consumption of these regions. Figures $7 \mathrm{a}-7 \mathrm{c}$ show the LES results obtained with the grid resolution of 600 cells in HRL in the present work. These figures show the progress variable for the duration between the collision of the triple point with the upper and lower walls. Clearly, there is a very good qualitative agreement between these figures and the experimental results of Kiyanda [62] shown in Fig. 6. Although the experiment shown in Fig. $\underline{6}$ corresponds to an activation energy higher than that considered in the present work, both mixtures show irregular structures with a turbulent reaction zone. Figure 7a shows that due to the collision of the triple point with the upper wall an unburned gas pocket has detached from the shock front. When the triple point moves downward the unburned pocket shrinks such that before its collision with the bottom wall the pocket has been fully consumed. The present numerical results show that the unburned pocket remains behind the front for about five HRL, which is in agreement with the experimental observations of Radulescu [15]. Furthermore, according to the schlieren image shown in Fig. 6c before the collision of the triple point with the wall, the traverse wave collides with the bottom wall at point $\mathrm{N}$. This results in the formation of a reflected wave (labelled R.W in the figure) from the wall. Referring again to Fig. 3c, it is deduced that the LES can predict this

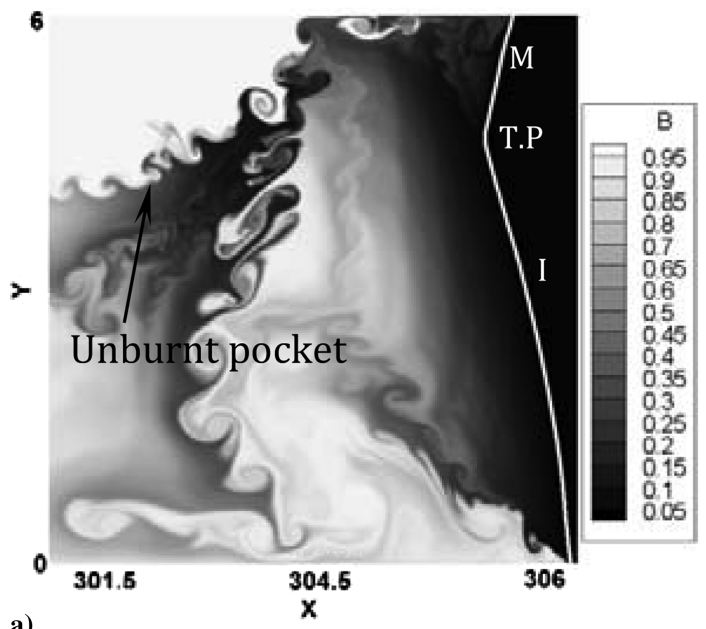

a)

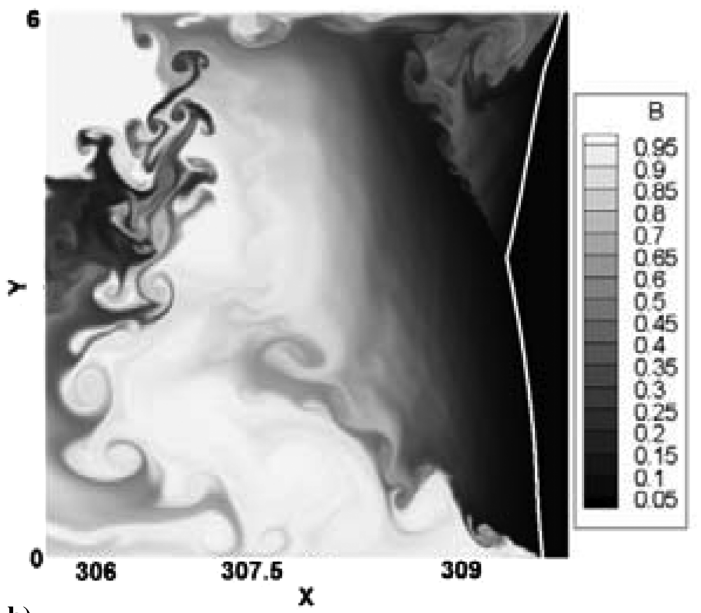

b)

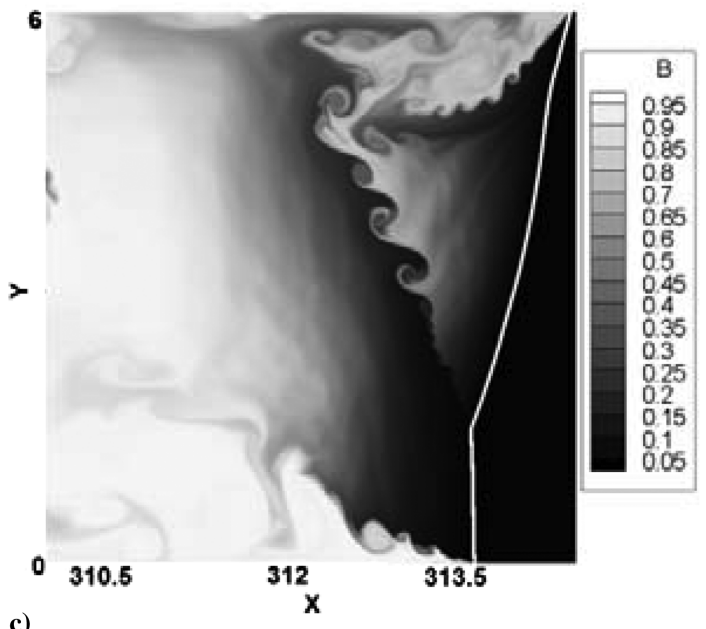

c)

Fig. 7 LES of the present work for a resolution of 600 cells per HRL.

scenario. Such a feature has not been observed in Euler or NS simulations (Figs. $\underline{3 a}$ and $\underline{3 b}$ ). Figure $\underline{6}$ does not show the vortical structures along the shear layer produced by $\mathrm{KH}$ instabilities. However, the KH unstable shear layer has been observed previously in schlieren images of Radulescu et al. [4], Bhattacharjee et al. [14], and Bhattacharjee [13].

Figure 8 illustrates the observations of the novel experiments performed recently by Bhattacharjee [13] in a methane-oxygen mixture. The frames clearly show the formation, evolution, and growth of jet flows after collision of two triple points. It can be seen that after collision of two triple points a pair of forward and backward jets is created. The backward jet moves into the hot product and is 

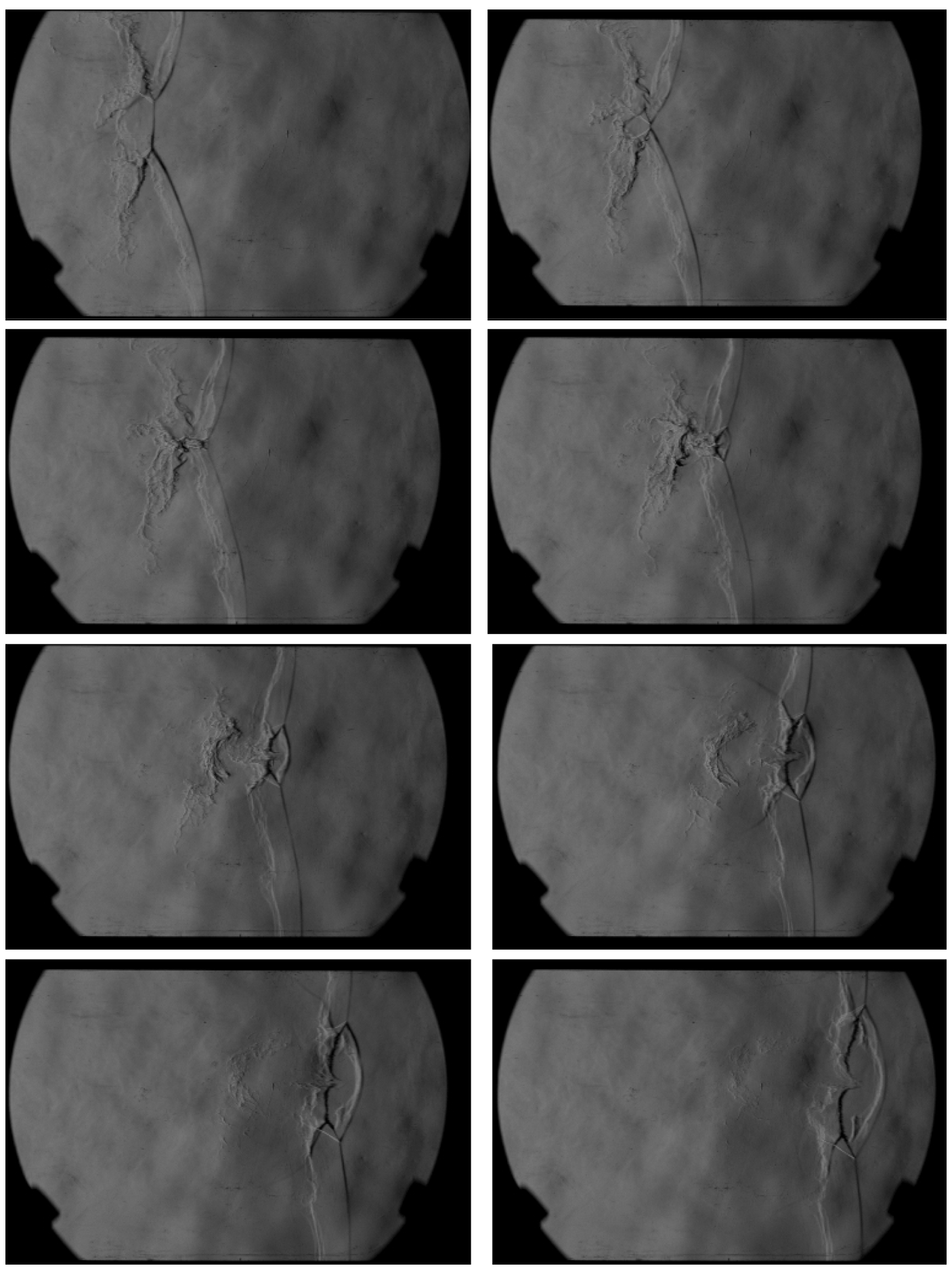

Fig. 8 Schlieren of the reaction zone structure of the detonation [13].

consumed shortly. The size of the forward jet, however, increases as it moves forward. The interaction of the forward jet with the shock front causes a bulge on the lead shock. It is also visible that after collision of the triple points pockets of unreacted gas detach from the shock front and recede from it. The small scale $\mathrm{KH}$ instabilities along the detached pocket are clearly visible in the third row of Fig. $\underline{8}$. The present LES results are in excellent agreement with the experimental results [13]. The jet flows, $\mathrm{KH}$ vortices, and unreacted gas pockets are clearly visible in the LES simulations (see Fig. 2c). In particular, comparing Figs. $2 \mathrm{a}$ and $2 \mathrm{~b}$ with Figs. $\underline{6}$ and $\underline{8}$ reveals that the structures obtained by $\overline{\text { Euler }}$ and $\mathrm{NS}$ simulations are even qualitatively different than the experiment. This further clarifies the role of dissipation at SGSs on controlling the reaction zone in irregular detonation, which is not included in a NS simulation with a laminar diffusion coefficient. Therefore, ignoring turbulence diffusion (i.e., dissipation at small scales) in the simulation of irregular gaseous detonations results in the appearance of nonphysical structures. As discussed in the previous sections, this generates highly enlarged jets and unburned pockets behind the front.

\section{Three-Dimensional Effects}

The relation between the 2-D simulations and the 3-D nature of highly turbulent structures of real detonations is currently an open question [16]. Turbulence is mostly generated in detonations by RM instability via the baroclinic $\nabla p \times \nabla \rho$ vorticity generation mechanism, which occurs due to the interaction of the strong pressure waves with the high-density gradients $[63,64]$. This leads to vorticity generation on a large scale comparable to the scale of the system. Another source of turbulence on small scales is the KH instability, but 
it appears to be less significant than RM instabilities for the flows under consideration [24]. Radulescu et al. [4,15] suggested this mechanism as a source of turbulence behind the front in gaseous detonations. Such a type of turbulence (shock-flame interaction) has also been observed in both numerical and experimental studies of DDT phenomena (e.g., $[24,46,65-70])$. In their investigation of DDT, Oran and Gamezo [24] showed that the computations in two dimensions were in agreement with those in three dimensions and experiments. Gamezo et al. [65] pointed out that even though in 3-D simulations the accelerating flame became more wrinkled the overall flame development is dominated by RM instability. Hence, their 2-D and 3-D simulations showed very similar results. The behavior of RM instability has been isolated and studied by many authors (e.g., $[63,71-73])$. The numerical study of RM instability by Anuchina et al. [74] showed that the growth rate of perturbations in a 3D case was higher than that in a 2D case at the identical initial amplitudes of perturbation and wavelengths. Li and Zhang [73] used both theoretical analysis and high-resolution numerical simulation to compare 2-D and 3-D growth rates of the RM instability. In the nonlinear regime, the growth rate in three dimensions was about $20 \%$ larger and $25 \%$ faster than that in two space dimensions. However, in the linear regime the growth rates of the instability in two and three dimensions were the same.

In the present problem, the RM instability tends to be the dominant mechanism generating turbulent structures behind the shock front. It is therefore speculated that the 2-D computations are in qualitative agreement with those in three dimensions and experiments. However, to determine the precise consumption time of the unreacted pockets, 3-D computations and complex chemical reaction mechanisms with more realistic SGS modeling for the reaction zone would both be needed.

\section{Conclusions}

The structure of irregular detonation with a turbulent reaction zone was studied with high-resolution (i.e., 600 cells per HRL) LES. The results were compared with those of Euler and NS simulations. This showed that hydrodynamic instabilities as described by the Euler and NS equations alone are not sufficient to result in the consumption of the unburned gases behind the main shock. Hence, the effect of vorticity generation and energy dissipation at SGSs plays an essential role in burning the unburned gases in this region. Therefore, ignoring the effect of dissipation at SGSs results in a nonphysical detonation structure. This can include large jet flow and a long tongue unburned pocket behind the main shock. Resolution analysis reveals that the structures obtained by Euler and NS solutions were very similar for the resolutions of 250 to 600 cells per HRL. Under low grid resolution, the LES solution showed the long tongue pocket behind the main shock. In a high-resolution situation of 600 cells per HRL, however, the tongue pocket disappeared. This demonstrates that in low grid resolution the numerical diffusion is high but is not high enough to result in the consumption of the unburned gas pockets. Therefore, solving the NS equations with the inclusion of the laminar diffusive coefficient leads to a nonphysical detonation structure. A proper turbulence modeling, e.g., compressible LES with very high resolution of at least 600 cells per HRL, is required to take into account the effect of dissipation on the small scale structures.

\section{Acknowledgments}

The first author is most grateful to Kiumars Mazaheri of Tarbiat Modares University and Matei Radulescu of University of Ottawa for their constructive comments over the course of this work.

\section{References}

[1] Lee, J. H. S., The Detonation Phenomenon, Cambridge Univ. Press, New York, NY, 2008.

[2] Voitsekhovsky, B. V., Mitrofanov, V. V., and Topchian, M. E., "Structure of a Detonation Front in Gases," Izvestiva Akademii Nauk USSR, 1963.

[3] Mahmoudi, Y., and Mazaheri, K., "Triple Point Collisions and Hot Spots in Detonations with Regular Structure," Combustion Science and
Technology, Vol. 184, Nos. 7-8, 2012, pp. 1135-1151. doi:10.1080/00102202.2012.664004

[4] Radulescu, M. I., Sharpe, G. J., Lee, J. H. S., Kiyanda, C. B., Higgins, A. J., and Hanson, R. K., "The Ignition Mechanism in Irregular Structure Gaseous Detonations," Proceedings of the Combustion Institute, Vol. 30, No. 2, 2005, pp. 1859-1867. doi:10.1016/j.proci.2004.08.047

[5] Austin, J. M., "The Role of Instability in Gaseous Detonation," Ph.D. Dissertation, California Inst. of Technology, Pasadena, CA, 2003.

[6] Oran, E. S., Young, T. R., Boris, J. P., Picone, J. M., and Edwards, D. H., "A Study of Detonation Structure: The Formation of Unreacted Gas Pockets," Symposium (International) on Combustion, Vol. 19, No. 1, 1982, pp. 573-582. doi:10.1016/S0082-0784(82)80231-2

[7] Radulescu, M. I., "The Propagation and Failure Mechanism of Gaseous Detonations: Experiments in Porous-Walled Tubes," Ph.D. Dissertation, McGill Univ., Montreal, 2003.

[8] Sabzpooshani, M., and Mazaheri, K., "Formation of Unburnt Pockets in Gaseous Detonation," Combustion, Explosion, and Shock Waves, Vol. 45, No. 2, 2009, pp. 182-189. doi:10.1007/s10573-009-0024-6

[9] Subbotin, V., "Two Kinds of Transverse Wave Structures in Multi-Front Detonation," Fizika Goreniya i Vzryva, Vol. 11, No. 1, 1975, pp. 96102. doi:10.1007/BF00742862

[10] Gamezo, V. N., Desbordes, D., and Oran, E. S., "Formation and Evolution of Two-Dimensional Cellular Detonations," Combustion and Flame, Vol. 116, Nos. 1-2, 1999, pp. 154-165. doi:10.1016/S0010-2180(98)00031-5

[11] Oran, E. S., Weber, J. R., Stefaniw, E. I., Lefebvre, M. H., and Anderson, J. D., "A Numerical Study of Two-Dimensional H2-O2-Ar Detonation Using a Detailed Chemical Reaction Model," Combustion and Flame, Vol. 113, Nos. 1-2, 1998, pp. 147-163. doi:10.1016/S0010-2180(97)00218-6

[12] Mahmoudi, Y., and Mazaheri, K., "High Resolution Numerical Simulation of the Structure of 2-D Gaseous Detonations," Proceedings of the Combustion Institute, Vol. 33, No. 2, 2010, pp. 2187-2194. doi:10.1016/j.proci.2010.07.083

[13] Bhattacharjee, R., "Experimental Investigation of Detonation ReInitiation Mechanisms Following a Mach Reflection of a Quenched Detonation," M.A. Sc. Thesis, Univ. of Ottawa, Ottawa, 2013.

[14] Bhattacharjee, R., Lau-Chapdelaine, S. S. M., Maines, G., Maley, L., and Radulescu, M. I., "Detonation Re-Initiation Mechanism Following the Mach Refection of a Quenched Detonation," Proceedings of the Combustion Institute, Vol. 34, No. 2, 2013, pp. 1893-1901. doi:10.1016/j.proci.2012.07.063

[15] Radulescu, M. I., Sharpe, G. J., Law, C. K., and Lee, J. H. S., "The Hydrodynamic Structure of Unstable Cellular Detonations," Journal of Fluid Mechanics, Vol. 580, June 2007, pp. 31-81. doi:10.1017/S0022112007005046

[16] Mazaheri, K., Mahmoudi, Y., and Radulescu, M. I., "Diffusion and Hydrodynamic Instabilities in Gaseous Detonations," Combustion and Flame, Vol. 156, No. 6, 2012, pp. 2138-2154. doi:10.1016/j.combustflame.2012.01.024

[17] Mahmoudi, Y., Mazaheri, K., and Parvar, S., "Hydrodynamic Instabilities and Transverse Waves in Propagation Mechanism of Gaseous Detonations," Acta Astronautica, Vol. 91, 2013, 263-282. doi:10.1016/j.actaastro.2013.06.009

[18] Lee, J. H. S., "The Propagation Mechanism of Cellular Detonation," Proceedings of the 24th International Symposium on Shock Waves 1, 2004, pp. 19-30.

[19] Shepherd, J. E., "Detonation in Gases," Proceedings of the Combustion Institute, Vol. 32, 2009, pp. 83-98.

[20] Massa, L., Austin, J. M., and Jackson, T. L., "Triple-Point Shear Layers in Gaseous Detonation Waves," Journal of Fluid Mechanics, Vol. 586, Sept. 2007, pp. 205-248. doi: $10.1017 / \mathrm{S} 0022112007007008$

[21] Radulescu, M. I., and Lee, J. H. S., "The Failure Mechanism of Gaseous Detonations," Combustion and Flame, Vol. 131, Nos. 1-2, 2002, pp. $29-46$. doi:10.1016/S0010-2180(02)00390-5

[22] Mach, P., and Radulescu, M. I., "Mach Reflection Bifurcations as a Mechanism of Cell Multiplication in Gaseous Detonations," Proceedings of the Combustion Institute, Vol. 33, No. 2, 2010, pp. 2279-2285. doi:10.1016/j.proci.2010.06.145

[23] Williams, F. A., Combustion Theory, Benjamin-Cummings, Menlo Park, CA, 1985. 
[24] Oran, E. S., and Gamezo, V. N., "Origins of the Deflagration-toDetonation Transition in Gas-Phase Combustion," Combustion and Flame, Vol. 148, Nos. 1-2, 2007, pp. 4-47. doi:10.1016/j.combustflame.2006.07.010

[25] Poinsot, T., and Veynante, D., Theoretical and Numerical Combustion, 2nd ed., Edwards, 2005.

[26] Kirkpatrik, M. P., Armfield, S. W., Masri, A. R., and Ibrahim, S. S., "Large Eddy Simulation of a Propagating Turbulent Premixed Flame," Flow, Turbulence, and Combustion, Vol. 70, Nos. 1-4, 2003, pp. 1-19. doi:10.1023/B:APPL.0000004912.87854.35

[27] Peters, N., Turbulent Combustion, 1st ed., Cambridge Univ. Press, 2000.

[28] Yoshizawa, A., "Statistical Theory for Compressible Turbulent Shear Flows, with the Application to Subgrid Modeling," Physics of Fluids, Vol. 29, No. 7, 1986, pp. 2152-2164. doi: $10.1063 / 1.865552$

[29] Meyers, J., "Error-Landscape Assessment of Large-Eddy Simulations: A Review of the Methodology," Journal of Scientific Computing, Vol. 49, No. 1, 2011, pp. 65-77. doi:10.1007/s10915-010-9449-Z

[30] Germano, M., Piomelli, U., Moin, P., and Cabot, W. H., "A Dynamic Subgrid-Scale Eddy Viscosity Model," Physics of Fluids, Vol. 3, No. , 1991, pp. 1760-1765. doi:10.1063/1.857955

[31] Dauptain, A., Cuenot, B., and Poinsot, T. J., Large Eddy Simulation of a Supersonic Hydrogen-Air Diffusion Flame, Complex Effects in Large Eddy Simulation, Limassol, 2005.

[32] Sharpe, G. J., "Transverse Wave in Numerical Simulations of Cellular Detonation," Journal of Fluid Mechanics, Vol. 447, Nov. 2001, pp. 31-51. doi:10.1017/S0022112001005535

[33] Garnier, E., Adams, N., and Sagaut, P., Large Eddy Simulation for Compressible Flows, Springer-Verlag, New York, 2009.

[34] Aradjo, A., Ferreira, J. A., Oliveira, P., and Patricio, F., "The Use of Splitting Methods in the Numerical Simulation of Reacting Flows," Computing and Visualization in Science, Vol. 6, Nos. 2-3, 2004, pp. 5966.. doi:10.1007/BF02663034

[35] Mahmoudi, Y., and Mazaheri, K., "Operator Splitting in Simulation of Detonation Structure," 22nd International Colloquium on the Dynamics of Explosions and Reactive Systems, Minsk, Belarus, 2009.

[36] Spyropoulos, J. T., "A New Scheme for the Navier-Stokes Equations Employing Alternating-Direction Operator Splitting and Domain Decomposition,” Ph.D. Dissertation, Purdue Univ., Lafayette, IN, 1999.

[37] Colella, P., "Multidimensional Upwind Methods for Hyperbolic Conservation Laws," Journal of Computational Physics, Vol. 87, No. 1, 1990, pp. 171-200. doi:10.1016/0021-9991(90)90233-Q

[38] Saltzman, J., "An Unsplit 3D Upwind Method for Hyperbolic Conservation Laws," Journal of Computational Physics, Vol. 115, No. 1, 1994, pp. 153-168. doi:10.1006/jcph.1994.1184

[39] Berger, M. J., and Colella, P., "Local Adaptive Mesh Refinement for Shock Hydrodynamics," Journal of Computational Physics, Vol. 82, No. 1, 1989, pp. 64-84. doi:10.1016/0021-9991(89)90035-1

[40] Bourlioux, A., "Numerical Study of Unstable Detonations," Ph.D. Dissertation, Princeton Univ., Princeton, NJ, 1991.

[41] Mazaheri, K., "Mechanism of the Onset of Detonation in Blast Initiation," Ph.D. Dissertation, McGill Univ., Montreal, 1997.

[42] Han, Y., "Non-Reflecting Boundary Condition in Detonation Simulation," M.Sc. Thesis, Univ. of Calgary, Calgary, AB, Canada, 2003.

[43] Lee, J. H. S., and Radulescu, M. I., "On the Hydrodynamic Thickness of Cellular Detonations," Combustion, Explosion, and Shock Waves, Vol. 41, No. 6, 2005, pp. 745-765. doi:10.1007/s10573-005-0084-1

[44] Hwang, P., Fedkiw, R. P., Merriman, B., Aslam, T. D., Karagozian, A. R., and Oshre, S. J., "Numerical Resolution of Pulsating Detonation Waves," Combustion Theory and Modeling, Vol. 4, No. 3, 2000, pp. 217-240. doi:10.1088/1364-7830/4/3/301

[45] Kasimov, A. R., and Stewart, D. S., "On the Dynamics of Self-Sustained One-Dimensional Detonations: A Numerical Study in the ShockAttached Frame," Physics of Fluids, Vol. 16, No. 10, 2004, pp. 35663578 . doi:10.1063/1.1776531

[46] Gamezo, V. N., Khokhlov, A. M., and Oran, E. S., "The Influence of Shock Bifurcation on Shock Flame Interactions and DDT," Combustion and Flame, Vol. 126, No. 4, 2001, pp. 1810-1826. doi:10.1016/S0010-2180(01)00291-7

[47] Singh, S., Powers, J. M., and Paolucci, S., "Detonation Solutions from Reactive Navier-Stokes Equations," 37th AIAA Aerospace Sciences Meeting and Exhibit, Reno, NV, 1999.

[48] Deiterding, R., "Parallel Adaptive Simulation of Multi-Dimensional Detonation Structures," Ph.D. Dissertation, Technical Univ. of Cottbus, Cottbus, Germany, 2003.

[49] Hu, X. Y., Khoo, B. C., Zhang, D. L., and Jiang, Z. L., "The Cellular Structure of a Two-Dimensional $\mathrm{H}_{2} \mathrm{O}_{2}-\mathrm{Ar}$ Detonation Wave," Combustion Theory and Modeling, Vol. 8, No. 2, 2004, pp. 339-359. doi:10.1088/1364-7830/8/2/008

[50] Hu, X. Y., Zhang, D. L., Khoo, B. C., and Jiang, Z. L., "The Structure and Evolution of a Two-Dimensional $\mathrm{H}_{2} \mathrm{O}_{2} / \mathrm{Ar}$ Cellular Detonation," Shock Waves, Vol. 14, Nos. 1-2, 2005, pp. 37-44. doi:10.1007/s00193-004-0234-5

[51] Mahmoudi, Y., Mazaheri, K., and Radulescu, M. I., "Triple Points Collision in Unstable Detonations," 23rd International Colloquium on the Dynamics of Explosions and Reactive Systems, Irvine, CA, 2011.

[52] Mazaheri, K., Mahmoudi, Y., and Radulescu, M. I., "Diffusion in Gasous Detonations," 23rd International Colloquium on the Dynamics of Explosions and Reactive Systems, Irvine, CA, 2011.

[53] Sharpe, G. J., and Falle, S. A. E. G., "Two-Dimensional Numerical Simulations of Idealized Detonations," Proceedings of the Royal Society of London, Series A: Mathematical and Physical Sciences, Vol. 456, No. 2001, 2000, pp. 2081-2100.

doi:10.1098/rspa.2000.0603

[54] Austin, J. M., Pintgen, F., and Shepherd, J. E., "Reaction Zones in Highly Unstable Detonations," Proceedings of the Combustion Institute, Vol. 30, No. 2, 2005, pp. 1849-1857. doi:10.1016/j.proci.2004.08.157

[55] Edwards, D. H., Hooper, G., Job, E. M., and Parry, D. J., "The Behavior of Frontal and Transverse Shocks in Gaseous Detonation Waves," Astronautica Acta, Vol. 15, 1970, pp. 323-333.

[56] Pintgen, F., Eckett, C. A., Austin, J. M., and Shepherd, J. E., "Direct Observations of Reaction Zone Structure in Propagating Detonations," Combustion and Flame, Vol. 133, No. 3, 2003, pp. 211-229. doi:10.1016/S0010-2180(02)00458-3

[57] Gamezo, V. N., Desbordes, D., and Oran, E. S., "Two-Dimensional Reactive Flow Dynamics in Cellular Detonation Waves," Shock Waves, Vol. 9, No. 1, 1999, pp. 11-17. doi:10.1007/s001930050134

[58] Ng, H. D., Higgins, A. J., Kiyanda, C. B., Radulescu, M. I., Lee, J. H. S., Bates, K. R., and Nikiforakis, N., "Nonlinear Dynamics and Chaos Analysis of One-Dimensional Pulsating Detonations," Combustion Theory and Modeling, Vol. 9, No. 1, 2005, pp. 159-170. doi:10.1080/13647830500098357

[59] Nikolic, M., Williams, D. N., and Bauwens, L., "Detonation Cell Sizes: A Numerical Study," AIAA Paper 1999-0967, 1999.

[60] Henrick, A. K., Aslam, T. D., and Powers, J. M., "Simulations of Pulsating One-Dimensional Detonations with True Fifth Order Accuracy," Journal of Computational Physics, Vol. 213, No. 1, 2006, pp. 311-329. doi:10.1016/j.jcp.2005.08.013

[61] Radulescu, M. I., Papi, A., and Quirk, J. J., "The Origin of Shock Bifurcations in Cellular Detonations," 22nd International Colloquium on the Dynamics of Explosions and Reactive Systems, Minsk, Belarus, 2009.

[62] Kiyanda, C. B.., "Photographic Study of the Structure of Irregular Detonation Waves," M.Sc. Thesis, McGill Univ., Montreal, 2005.

[63] Brouillette, M., "The Richtmyer-Meshkov Instability," Annual Review of Fluid Mechanics, Vol. 34, 2002, pp. 445-468. doi:10.1146/annurev.fluid.34.090101.162238

[64] Lombardini, M., Deiterding, R., and Pullin, D. I., "Large-Eddy Simulation of the Richtmyer-Meshkov Instability in a Converging Geometry," Proceedings of QLES 2007 International Symposium, Springer, Leuven, The Netherlands, 2008

[65] Gamezo, V. N., Ogawa, T., and Oran, E. S., "Numerical Simulations of Flame Propagation and DDT in Obstructed Channels Filled with Hydrogen-Air Mixture," Proceedings of the Combustion Institute, Vol. 31, No. 2, 2007, pp. 2463-2471. doi:10.1016/i.proci.2006.07.220

[66] Gamezo, V. N., Ogawa, T., and Oran, E. S., "Flame Acceleration and DDT in Channels with Obstacles: Effect of Obstacle Spacing," Combustion and Flame, Vol. 155, Nos. 1-2, 2008, pp. 302-315. doi:10.1016/i.combustflame.2008.06.004

[67] Khokhlov, A. M., Oran, E. S., and Thomas, G. O., "Numerical Simulation of Deflagration-to-Detonation Transition: The Role of Shock-Flame Interactions in Turbulent Flames," Combustion and 
Flame, Vol. 117, Nos. 1-2, 1999, pp. 323-339. doi:10.1016/S0010-2180(98)00076-5

[68] Khokhlov, A. M., Oran, E. S., Chtchelkanova, A. Y., and Wheeler, J. C., "Interaction of a Shock with a Sinusoidally Perturbed Flame," Combustion and Flame, Vol. 117, Nos. 1-2, 1999, pp. 99-116. doi:10.1016/S0010-2180(98)00090-X

[69] Khokhlov, A. M., Austin, J. M., Pintgen, F., and Shepherd, J. E., "Numerical Study of the Detonation Wave Structure in EthyleneOxygen Mixtures," 42th AIAA Aerospace Sciences Meeting and Exhibit, Reno, NV, 2004.

[70] Thomas, G. O., Bambrey, R., and Brown, C., "Experimental Observations of Turbulent Combustion and Transition to Detonation Following Shock-Flame Interaction," Combustion Theory and Modelling, Vol. 5, No. 4, 2001, pp. 573-594. doi:10.1088/1364-7830/5/4/304

[71] Cloutman, L. D., and Wehner, M. F., "Numerical Simulation of Richtmyer-Meshkov Instabilities," Physics of Fluids, Vol. 4, No., 1992, pp. 1821-1830. doi: $10.1063 / 1.858403$
[72] Lebo, I. G., Rozanov, V. B., Tishkin, V. F., Nikishin, V. V., Popov, I. V., and Favorsky, A. P., "Numerical Simulation of Richtmyer-Meshkov Instability," 5th International Workshop on Compressible Turbulent Mixing, Stony Brook, NY, 1995.

[73] Li, X. L., and Zhang, Q., "A Comparative Numerical Study of the Richtmyer-Meshkovinstability with Nonlinear Analysis in Two and Three Dimensions," Physics of Fluids, Vol. 9, No. 10, 1997, pp. 3069 3077. doi: $10.1063 / 1.869415$

[74] Anuchina, N. N., Volkov, V. I., Gordeychuk, V. A., Es'kov, N. S., Ilyutina, O. S., and Kozyrev, O. M., "Numerical Simulations of Rayleigh-Taylor and Richtmyer-Meshkovinstability Using MAH-3 Code," Journal of Computational and Applied Mathematics, Vol. 168, Nos. 1-2, 2004, pp. 11-20. doi:10.1016/j.cam.2003.06.008

J. Oefelein Associate Editor 


\section{Queries}

1. AU: As per style, article title should be 12 words. Please check and confirm the edit made in the title.

2. AU: Please list postal code in the affiliation line for the author "S. Emami".

3. AU: For each author, please provide the following information to be included in the first-page footnotes: title/position, department, e-mail address (optional), and AIAA membership status (if applicable).

4. AU: Please note that the abstract has been edited to be in the third person, per journal guidelines. Please check that your meaning was retained.

5. AU: Please check that the copyright (C) type is correct. Please note that the code will be added upon publication.

6. AU: The sentence beginning "Unlike classical turbulence" was changed for clarity: please check that meaning was not altered.

7. AU: The sentence beginning "Compressible flows" was changed for clarity. Please check that meaning was not altered.

8. AU: The sentence beginning "The SGS stress tensor" was changed for clarity. Please check that meaning was not altered.

9. AU: The sentence beginning "This model" was changed for clarity. Please check that meaning was not altered.

10. AU: Note that hrl was changed to HRL per style for acronyms throughout. Please check that meaning was altered.

11. AU: Headings were added to Table 1 per style. Please check that they are acceptable.

12. AU: The sentence beginning "However, in mixtures" was changed for clarity. Please check that meaning was not altered.

13. AU: AIAA has not indicated color use for your paper. Please confirm that your paper should be grayscale and that all figures are satisfactory. If any replacement figures are needed, please send them in .eps or.tiff format to aiaa-proofs@ beacon.com and use code B34986 in the subject line. Formats of .jpg, .doc, or .pdf can be used with some loss of quality.

14. AU: Please check that Figs. 6 and 7 are labeled a-c properly.

15. AU: Please list page range for Refs. $[1,2,17,23,25,27,31,33]$.

16. AU: Please list volume number, issue number, full journal title, date, and page range for Ref. [2].

17. AU: Please check the edit(s) made in Ref. [6].

18. AU: Please check that "M.A. Sc. Thesis" is correct degree in Ref. [13].

19. AU: Please check the edit(s) made in Ref. [17].

20. AU: If Refs. $[18,35,47,51,52,61,64,69,72]$ are published proceedings, please provide the names and locations of the publishers (NOT of the conference hosts) and the page range. If they are conference papers, please provide the paper numbers and the organizers' names. If they are CD-ROMs, please provide the names and locations of the CD-ROM producers.

21. AU: Please provide the issue number for Ref. [19]. 
22. AU: Please list publisher location for Refs. [25, 27, 31].

23. AU: Please provide the issue number for Ref. [55]. 